\title{
Analysis and Numerical Simulation of Hyperbolic Shallow Water Moment Equations
}

\author{
Julian Koellermeier ${ }^{1,2, *}$ and Marvin Rominger ${ }^{1}$ \\ ${ }^{1}$ Institut für Mathematik, Freie Universität Berlin, Berlin, Germany. \\ ${ }^{2}$ School of Mathematical Sciences, Peking University, Beijing, China.
}

Received 24 April 2019; Accepted (in revised version) 17 January 2020

\begin{abstract}
Shallow Water Moment Equations allow for vertical changes in the horizontal velocity, so that complex shallow flows can be described accurately. However, we show that these models lack global hyperbolicity and the loss of hyperbolicity already occurs for small deviations from equilibrium. This leads to instabilities in a numerical test case. We then derive new Hyperbolic Shallow Water Moment Equations based on a modification of the system matrix. The model can be written in analytical form and hyperbolicity can be proven for a large number of equations. A second variant of this model is obtained by generalizing the modification with the help of additional parameters. Numerical tests of a smooth periodic problem and a dam break problem using the new models yield accurate and fast solutions while guaranteeing hyperbolicity.
\end{abstract}

AMS subject classifications: 76D05, 35L65, 65M08

Key words: Shallow water equation, moment method, hyperbolicity.

\section{Introduction}

The shallow water equations (SWE) are widely used to describe flows where the vertical velocity components are much smaller than the horizontal components. Apart from hydrodynamic applications such as the computation of currents in Venice bay [32], the shallow water equations are used in many other scientific fields like weather forecasts [8], gravitational flow models like snow avalanches [7], and granular flows of liquids in the field of chemical engineering [9].

Nevertheless, the shallow water equations are often limited in their applications due to the loss of information by averaging the vertical velocity, see [18]. The assumption of a constant horizontal velocity with respect to height is a strong simplification and has been shown to be inaccurate in measurements [20,33].

*Corresponding author. Email addresses: koellermeier@zedat.fu-berlin.de (J. Koellermeier), marvin.rominger@gmail.com (M. Rominger) 
A first approach to circumvent this problem is the multi-layer shallow water model, which yields a piecewise constant velocity profile to model changes in vertical direction, see e.g. $[5,14,28,35]$ for details on the model derivation, analysis, and numerical implementation. However, this introduces a number of difficulties, for example the coupling at the interfaces and hyperbolicity issues due to the discontinuous velocity description.

This paper investigates moment models for the shallow water equations, in which vertical changes of the velocity variable are allowed by adding further variables (socalled moments) such that deviations from that averaged constant velocity can be represented by a continuous polynomial. The moment method is already widely known in kinetic theory after the seminal work by H. Grad [16] and was later applied in channel flows [15,37] and debris flows [26].

In [27] a moment method for the shallow water equations was introduced that succeeds in describing linear and quadratic velocity profiles for a smooth test case. We will use this model called Shallow Water Moment Equations (SWME) as a starting point of our investigations.

However, similar to the moment method in kinetic theory $[2,24,39]$, hyperbolicity of the SWME is limited to a bounded domain in phase space. This lack of hyperbolicity was not discussed in detail in the shallow water moment model [27]. However, problems with hyperbolicity often lead to instabilities in other models, so that new hyperbolic models needed to be derived, for example $[3,31,34,38]$. For rarefied gases the lack of hyperbolicity could be overcome using several strategies $[4,13,22,23]$, effectively resulting in the change of some terms in the higher order equations, but up to now no strategy to derive hyperbolic shallow water moment equations exists.

The aim of the paper is to investigate the hyperbolicity of the shallow water moment equations and systematically derive modified systems of equations that yield global hyperbolicity and sufficient accuracy in numerical simulations.

We first focus on the original SWME system and prove that loss of hyperbolicity occurs in practical applications, even when starting from initial conditions that are inside the hyperbolicity region. This might result in unpredictable behavior of the solution and makes it difficult to justify the use of the standard model. In one example we show emerging instabilities in a numerical solution.

The hyperbolic regularization is then based on a linearization of the SWME model around a linear deviation from equilibrium, similar to the models in [2] and [23]. The resulting system of equations is called Hyperbolic Shallow Water Moment Equations (HSWME). We investigate the structure and explicit terms in detail to allow for a global hyperbolicity proof for the high order system. Using the insights of this proof, it is possible to generalize the approach so that predefined propagation speeds can be obtained. This is achieved by the addition of some parameters $\beta_{i}$ in the very last row of the system matrix, leading to a new hyperbolic system, called $\beta$-HSWME. The parameters are chosen so that the characteristic polynomial of the system matrix matches a given target polynomial. We show that there exists a unique solution and we give a practical example in which the solution is particularly simple to compute and only a single entry has to be 
changed in the matrix.

After analyzing linear stability of both new systems, we perform numerical tests for smooth and discontinuous initial data to access the accuracy of the hyperbolic regularizations. Despite the simplifications and changes in the system matrices, both new models show good agreement with a reference solution and the original SWME, derived in [27].

The remaining part of this paper is structured as follows: A brief summary of the shallow water moment equations and the lack of hyperbolicity will be presented in Section 2 together with an example for the breakdown of hyperbolicity. In Section 3 the two new hyperbolic shallow water moment models HSWME and $\beta$-HSWME will be presented and investigated, including a linear stability analysis. A numerical test case showcasing the stability improvement and a comparison using a smooth transport test case and a discontinuous dam break test case will be presented in Section 4. The paper ends with a short conclusion.

\section{Shallow Water Moment Model}

In this section the main ideas for the derivation of the one-dimensional shallow water moment model from [27] are briefly discussed, before we recall the general form of the hierarchical moment model. We show the lack of hyperbolicity and give an example for the breakdown of hyperbolicity during a simulation.

Starting from the incompressible Navier-Stokes equations, the classical shallow water equations can be derived by averaging over the vertical variable assuming constant velocity in the same direction. In $1 \mathrm{D}$ this leads to the following evolution equations for water height $h$ and mean velocity $u_{m}$

$$
\begin{aligned}
& \partial_{t} h+\partial_{x}\left(h u_{m}\right)=0, \\
& \partial_{t}\left(h u_{m}\right)+\partial_{x}\left(h u_{m}^{2}+\frac{1}{2} g h^{2}\right)=-\frac{v}{\lambda} u_{m}
\end{aligned}
$$

where $v$ is the friction coefficient, $g$ is the gravitation constant and $\lambda$ is the slip length, see [27].

A drawback of the shallow water equations is that the velocity is usually constant and vertical variations in the velocity cannot be represented, except for models which add so called shape factors $[19,25,29]$. However, these shape factors typically only allow for a rather fixed parametrization. In [27], a new model for shallow flows is derived, based on two main ideas. The first main idea is introducing a scaled vertical variable $\zeta(t, x)$ using

$$
\zeta(t, x):=\frac{z-h_{b}(t, x)}{h_{s}(t, x)-h_{b}(t, x)}=\frac{z-h_{b}(t, x)}{h(t, x)},
$$

with $h(t, x)=h_{s}(t, x)-h_{b}(t, x)$ the water height from the bottom $h_{b}$ to the surface $h_{s}$, so that $\zeta:[0, T] \times \mathbb{R} \rightarrow[0,1]$. This transforms the $z$-direction from a physical space (see red lines in Fig. 1) to a projected space (see blue lines in Fig. 1). 
The transformation of the vertical variable leads to the following transformed shallow water model when assuming a non-constant velocity profile $u$

$$
\begin{aligned}
& \partial_{t} h+\partial_{x}\left(h u_{m}\right)=0, \\
& \partial_{t}\left(h u_{m}\right)+\partial_{x}\left(h u^{2}+\frac{g}{2} h^{2}\right)-\partial_{\zeta}\left(\frac{v}{h} \partial_{\zeta} u\right)+\partial_{\zeta}(h u \omega)=-h g \frac{\partial h_{b}}{\partial x},
\end{aligned}
$$

where the vertical coupling term $\omega$ in $1 \mathrm{D}$ is given by

$$
h \omega:=\int_{0}^{1} \partial_{x}\left(h \int_{0}^{\zeta} u_{m}-u d \tilde{\zeta}\right) .
$$

The second main idea from [27] is the moment expansion of the velocity variable, used for expressing more complex velocities e.g. linear, quadratic or cubic in the transformed vertical direction. We thus expand $u:[0, T] \times \mathbb{R} \times[0,1] \rightarrow \mathbb{R}$ as

$$
u(t, x, \zeta)=u_{m}(t, x)+\sum_{j=1}^{N} \alpha_{j}(t, x) \phi_{j}(\zeta),
$$

where $u_{m}:[0, T] \times \mathbb{R} \rightarrow \mathbb{R}$ is again the mean velocity, $\phi_{j}:[0,1] \rightarrow \mathbb{R}$ are the scaled Legendre polynomials of degree $j$ and the $\alpha_{j}:[0, T] \times \mathbb{R} \rightarrow \mathbb{R}$ with $j \in[1,2, \cdots, N]$ are corresponding basis coefficients at time $t$ and position $x$, also called moments. These coefficients will give rise to different horizontal velocities over the height, which is an extension compared to the classical shallow flow equation where the horizontal velocity does not change over the height, see Fig. $1 . N \in \mathbb{N}$ denotes the order of the velocity expansion and the maximal degree of the Legendre polynomials.

Instead of the classical shallow water system for water height $h$ and mean velocity $u_{m}, N$ additional coefficients $\alpha_{j}$ need to be computed, so that $N$ further equations are required. Note that for the case $N=0$ there are no further equations and the velocity is equal to the mean velocity, i.e. $u(t, x, \zeta)=u_{m}(t, x)$, so that the shallow water equations (2.1) and (2.2) are obtained from the transformed equations (2.3) and (2.4).

Additional $N$ equations are derived by projecting the momentum equation (2.4) onto Legendre polynomials, i.e. multiplication of Eq. (2.4) with $\phi_{j}(\zeta)$ for $j=1, \cdots, N$ and subsequent integration with respect to $\zeta$.

The detailed derivation is given in [27]. Here, we state the resulting $i^{t h}$-moment equation in $1 \mathrm{D}$ with $i \in\{1, \cdots, N\}$ :

$$
\frac{\partial\left(h \alpha_{i}\right)}{\partial t}+\frac{\partial \tilde{F}_{i}}{\partial x}=\tilde{Q}_{i}+P_{i}
$$

with the conservative fluxes

$$
\tilde{F}_{i}=h\left(2 u_{m} \alpha_{i}+\sum_{j, k=1}^{N}(2 i+1) \int_{0}^{1} \phi_{i} \phi_{j} \phi_{k} \mathrm{~d} \zeta \alpha_{j} \alpha_{k}\right),
$$




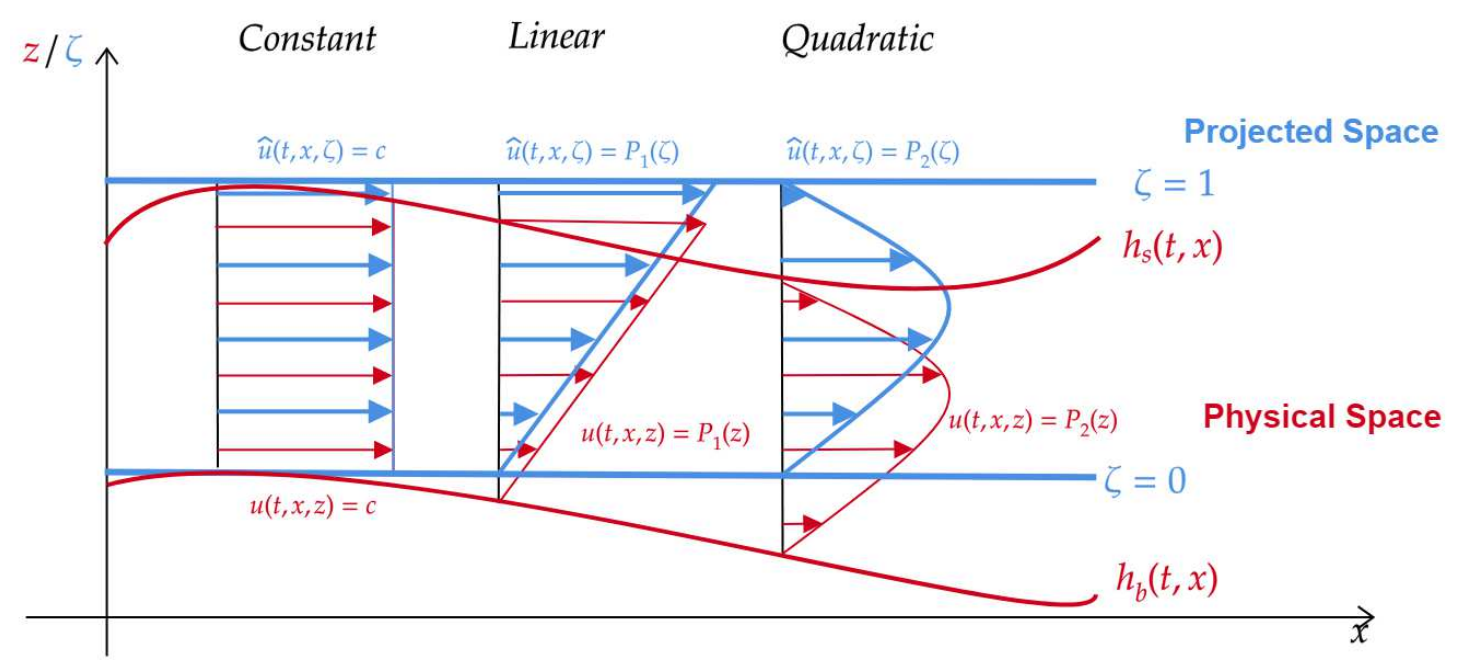

Figure 1: Different velocity profiles for polynomials $P_{N}(z)$ or $P_{N}(\zeta)$ with $N=0$ for constant velocity, $N=1$ for linear velocity and $N=2$ for quadratic velocity. The mapping of physical vertical space (red) to projected vertical unit space (blue) is depicted.

the non-conservative terms

$$
\tilde{Q}_{i}=u_{m} \frac{\partial\left(h \alpha_{i}\right)}{\partial x}-\sum_{j, k=1}^{N}(2 i+1) \int_{0}^{1} \phi_{i}^{\prime}\left(\int_{0}^{\zeta} \phi_{j} \mathrm{~d} \hat{\zeta}\right) \phi_{k} \mathrm{~d} \zeta \cdot \alpha_{k} \frac{\partial\left(h \alpha_{j}\right)}{\partial x}
$$

and the right-hand side (friction) source terms

$$
P_{i}=-(2 i+1) \frac{v}{\lambda}\left(u_{m}+\sum_{j=1}^{N}\left(1+\frac{\lambda}{h} \int_{0}^{1} \phi_{i}^{\prime} \phi_{j}^{\prime} \mathrm{d} \zeta\right) \alpha_{j}\right) .
$$

This leads to a closed shallow water moment system with a total of $N+2$ coupled equations for a $1 D$-problem, compared to only two equations for the shallow water equations:

$$
\underbrace{1}_{\text {mass balance equation }}+\underbrace{1}_{\text {momentum balance equation }}+\underbrace{N}_{\text {moment equations }}=N+2 .
$$

The complete system of equations can be rewritten in the form

$$
\frac{\partial V}{\partial t}+\frac{\partial F(V)}{\partial x}=Q(V) \frac{\partial V}{\partial x}+P(V)
$$

with variable vector $V=\left(h, h u, h \alpha_{1}, \cdots, h \alpha_{N}\right)^{T} \in \mathbb{R}^{N+2}, \frac{\partial F(V)}{\partial x}, P(V) \in \mathbb{R}^{N+2}$ and $Q(V) \in$ $\mathbb{R}^{(N+2) \times(N+2)}$. The matrix $Q(V)$ encodes the non-conservative contribution to the flux while the vector $\frac{\partial F(V)}{\partial x}$ contains the conservative flux terms. We use conservative variables $V$ to allow for easy exact conservation of mass and conservation of momentum in equilibrium with zero friction. 


\subsection{Hyperbolicity of the SWME}

For mathematical models of flow problems, especially in hydrodynamics but also for gas dynamics, hypersonic flows or particle dynamics, hyperbolicity of the equations is a paramount property $[3,30,31,38]$. It ensures the propagation of information with realvalued and bounded propagation speeds. Otherwise, non-physical oscillations could occur, leading to a breakdown of the solution. This behavior was observed in comparable models, e.g. moment models for rarefied gases [2,22].

We will use the following definition of hyperbolicity [12].

Definition 2.1. A system of first order partial differential equations of the form

$$
\frac{\partial U(t, x)}{\partial t}+A(U) \frac{\partial U(t, x)}{\partial x}=0 \quad \text { on }(0, \infty) \times \mathbb{R},
$$

with $U:[0, \infty) \times \mathbb{R} \rightarrow \mathbb{R}^{N}, U \in \mathbb{R}^{N}$, and $A:[0, \infty) \times \mathbb{R} \rightarrow \mathbb{R}^{N \times N}$ is globally hyperbolic if $A(U)$ is diagonalizable with real eigenvalues for all states $U$.

Remark 2.1. Definition 2.1 is automatically fulfilled if the matrix $A(U)$ has distinct real eigenvalues.

In the following, we recall the hyperbolicity investigation of the lower order SWME for $N=1,2,3$ from [27] and then take a closer look at the hyperbolicity loss for larger $N$.

\subsubsection{First order system}

Choosing $N=1$, i.e. linear velocity change with the vertical variable, the first order shallow water moment model reads

$$
\partial_{t}\left(\begin{array}{c}
h \\
h u_{m} \\
h \alpha_{1}
\end{array}\right)+\partial_{x}\left(\begin{array}{c}
h u_{m} \\
h u_{m}^{2}+g \frac{h^{2}}{2}+\frac{1}{3} h \alpha_{1}^{2} \\
2 h u_{m} \alpha_{1}
\end{array}\right)=Q \partial_{x}\left(\begin{array}{c}
h \\
h u_{m} \\
h \alpha_{1}
\end{array}\right)-\frac{v}{\lambda} P
$$

with

$Q=\left(\begin{array}{ccc}0 & 0 & 0 \\ 0 & 0 & 0 \\ 0 & 0 & u_{m}\end{array}\right), \quad P=\left(\begin{array}{c}0 \\ u_{m}+\alpha_{1} \\ 3\left(u_{m}+\alpha_{1}+4 \frac{\lambda}{h} \alpha_{1}\right)\end{array}\right) \quad$ and $\quad \frac{\partial F}{\partial V}=\left(\begin{array}{ccc}0 & 1 & 0 \\ -u_{m}^{2}-\frac{\alpha_{1}^{2}}{3}+g h & 2 u_{m} & \frac{2 \alpha_{1}}{3} \\ -2 u_{m} \alpha_{1} & 2 \alpha_{1} & u_{m}\end{array}\right)$,

leading to the system matrix

$$
A=\frac{\partial F}{\partial V}-Q=\left(\begin{array}{ccc}
0 & 1 & 0 \\
-u_{m}^{2}-\frac{\alpha_{1}^{2}}{3}+g h & 2 u_{m} & \frac{2 \alpha_{1}}{3} \\
-2 u_{m} \alpha_{1} & 2 \alpha_{1} & u_{m}
\end{array}\right)
$$

It is easy to show that the first order system has the distinct real eigenvalues

$$
\lambda_{1,2}=u_{m} \pm \sqrt{g h+\alpha_{1}^{2}} \text { and } \lambda_{3}=u_{m} .
$$

Due to $h>0$, the first order shallow water moment model is hyperbolic. 


\subsubsection{Second order system}

The second order moment model using $N=2$ is given by

$$
\partial_{t}\left(\begin{array}{c}
h \\
h u_{m} \\
h \alpha_{1} \\
h \alpha_{2}
\end{array}\right)+\partial_{x}\left(\begin{array}{c}
h u_{m} \\
h u_{m}^{2}+g \frac{h^{2}}{2}+\frac{1}{3} h \alpha_{1}^{2}+\frac{1}{5} h \alpha_{2}^{2} \\
2 h u_{m} \alpha_{1}+\frac{4}{5} h \alpha_{1} \alpha_{2} \\
2 h u_{m} \alpha_{2}+\frac{2}{3} h \alpha_{1}^{2}+\frac{2}{7} h \alpha_{2}^{2}
\end{array}\right)=Q \partial_{x}\left(\begin{array}{c}
h \\
h u_{m} \\
h \alpha_{1} \\
h \alpha_{2}
\end{array}\right)-\frac{v}{\lambda} P
$$

with

$$
Q=\left(\begin{array}{cccc}
0 & 0 & 0 & 0 \\
0 & 0 & 0 & 0 \\
0 & 0 & u_{m}-\frac{\alpha_{2}}{5} & \frac{\alpha_{1}}{5} \\
0 & 0 & \alpha_{1} & u_{m}+\frac{\alpha_{2}}{7}
\end{array}\right) \quad \text { and } \quad P=\left(\begin{array}{c}
0 \\
u_{m}+\alpha_{1}+\alpha_{2} \\
3\left(u_{m}+\alpha_{1}+\alpha_{2}+4 \frac{\lambda}{h} \alpha_{1}\right) \\
5\left(u_{m}+\alpha_{1}+\alpha_{2}+12 \frac{\lambda}{h} \alpha_{2}\right)
\end{array}\right)
$$

This leads to

$$
\frac{\partial F}{\partial V}=\left(\begin{array}{cccc}
0 & 1 & 0 & 0 \\
-\frac{\alpha_{1}^{2}}{3}-u_{m}^{2}+g h-\frac{\alpha_{2}^{2}}{5} & 2 u_{m} & \frac{2 \alpha_{1}}{3} & \frac{2 \alpha_{2}}{5} \\
-2 \alpha_{1} u_{m}-\frac{4}{5} \alpha_{1} \alpha_{2} & 2 \alpha_{1} & 2 u_{m}+\frac{4 \alpha_{2}}{5} & \frac{4 \alpha_{1}}{5} \\
-\frac{2}{3} \alpha_{1}^{2}-2 \alpha_{2} u_{m}-\frac{2}{7} \alpha_{2}^{2} & 2 \alpha_{2} & \frac{4 \alpha_{1}}{3} & 2 u_{m}+\frac{4 \alpha_{2}}{7}
\end{array}\right)
$$

and we get

$$
A=\frac{\partial F}{\partial V}-Q=\left(\begin{array}{cccc}
0 & 1 & 0 & 0 \\
-\frac{\alpha_{1}^{2}}{3}-u_{m}^{2}+g h-\frac{\alpha_{2}^{2}}{5} & 2 u_{m} & \frac{2 \alpha_{1}}{3} & \frac{2 \alpha_{2}}{5} \\
-2 \alpha_{1} u_{m}-\frac{4}{5} \alpha_{1} \alpha_{2} & 2 \alpha_{1} & u_{m}+\alpha_{2} & \frac{3 \alpha_{1}}{5} \\
-\frac{2}{3} \alpha_{1}^{2}-2 u_{m} \alpha_{2}-\frac{2}{7} \alpha_{2}^{2} & 2 \alpha_{2} & \frac{\alpha_{1}}{3} & u_{m}+\frac{3 \alpha_{2}}{7}
\end{array}\right)
$$

Scaling the coefficients $\alpha_{1}$ and $\alpha_{2}$ with $\frac{1}{\sqrt{g^{h}}}$, the four eigenvalues have the form

$$
\lambda_{i}=u_{m}+c_{i} \sqrt{g h} \quad \text { for } i=1,2,3,4,
$$

with $c_{i}$ being the $i^{\text {th }}$ root of

$$
\begin{aligned}
\chi(\lambda)= & \lambda^{4}-\frac{10}{7} \alpha_{2} \lambda^{3}-\left(1+\frac{6}{35} \alpha_{2}^{2}+\frac{6}{5} \alpha_{1}^{2}\right) \lambda^{2}+\left(\frac{22}{35} \alpha_{2}^{3}-\frac{6}{35} \alpha_{2} \alpha_{1}^{2}+\frac{10}{7} \alpha_{2}\right) \lambda \\
& -\frac{\alpha_{2}^{4}}{35}-\frac{6}{35} \alpha_{2}^{2} \alpha_{1}^{2}-\frac{3}{7} \alpha_{2}^{2}+\frac{\alpha_{1}^{4}}{5}+\frac{\alpha_{1}^{2}}{5} .
\end{aligned}
$$

Calculating the roots of this polynomial numerically for varying parameter values of $\alpha_{1}$ and $\alpha_{2}$, we see that they are complex numbers (see blue areas in Fig. 2(a)) leading to a lack of hyperbolicity. 


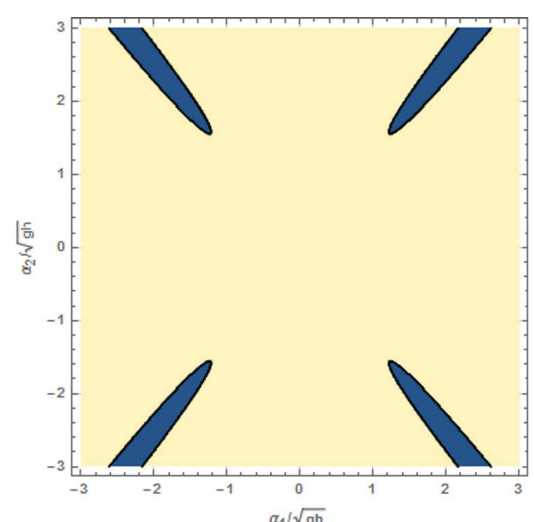

(a) $N=2$

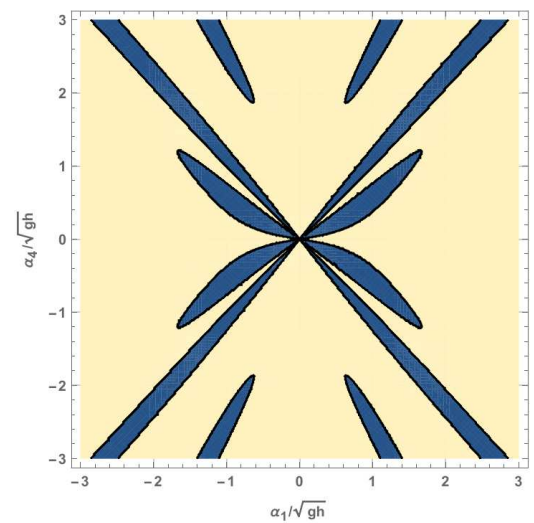

(c) $N=4$

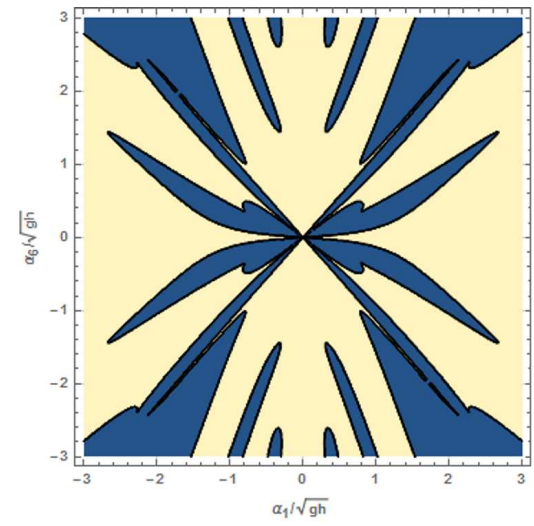

(e) $N=6$

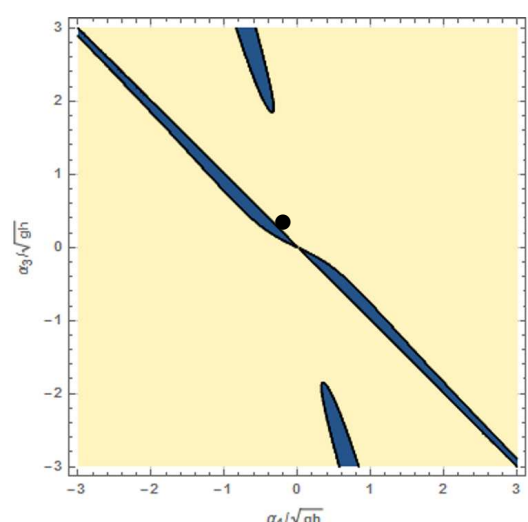

(b) $N=3$

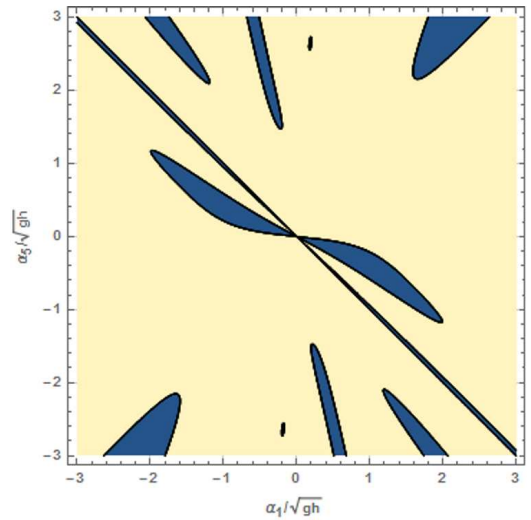

(d) $N=5$

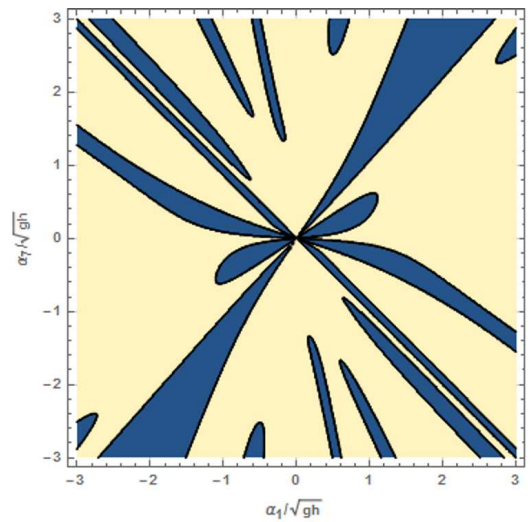

(f) $N=7$

Figure 2: Hyperbolicity breakdown (blue) and hyperbolic regions (beige) for different values of $\alpha_{1}$ and $\alpha_{N}$, $N=2,3,4,5,6,7$, respectively. Second order model (top left) and exemplary third order model at $\alpha_{2}=0$ (top right) reproduced from [27]. Initial condition for test case in Section 2.2 marked by "•" in 2(b). 


\subsubsection{Third order system}

The system matrix for the third order system can be derived from the third order system given in [27] analogously and reads

$$
\begin{aligned}
A & =\frac{\partial F}{\partial V}-Q \\
& =\left(\begin{array}{ccccc}
0 & 1 & 0 & 0 & 0 \\
-\frac{\alpha_{1}^{2}}{3}-u_{m}^{2}+g h-\frac{\alpha_{2}^{2}}{5}-\frac{\alpha_{3}^{2}}{7} & 2 u_{m} & \frac{2 \alpha_{1}}{3} & \frac{2 \alpha_{2}}{5} & \frac{2 \alpha_{3}}{7} \\
-\frac{2}{35}\left(9 \alpha_{3} \alpha_{2}+7 \alpha_{1}\left(5 u_{m}+2 \alpha_{2}\right)\right) & 2 \alpha_{1} & u_{m}+\alpha_{2} & \frac{3\left(\alpha_{1}+\alpha_{3}\right)}{5} & \frac{3 \alpha_{2}}{7} \\
-\frac{2}{21}\left(7 \alpha_{1}^{2}+9 \alpha_{1} \alpha_{3}+2 \alpha_{3}^{2}+3 \alpha_{2}\left(7 u_{m}+\alpha_{2}\right)\right) & 2 \alpha_{2} & \frac{\alpha_{1}}{3}+\frac{9 \alpha_{3}}{7} & u_{m}+\frac{3 \alpha_{2}}{7} & \frac{4 \alpha_{1}}{7}+\frac{\alpha_{3}}{3} \\
-\frac{2}{15}\left(15 u_{m} \alpha_{3}+4 \alpha_{2} \alpha_{3}+9 \alpha_{1} \alpha_{2}\right) & 2 \alpha_{3} & 0 & \frac{2\left(\alpha_{1}+\alpha_{3}\right)}{5} & u_{m}+\frac{\alpha_{2}}{3}
\end{array}\right) .
\end{aligned}
$$

The eigenvalues of $A$ again have the form $a_{i}=u_{m}+c_{i} \sqrt{g h}$ with $c_{i}$ being the $i^{\text {th }}$ root of a scaled polynomial given in the appendix of [27].

In Fig. 2(b), we exemplarily plot the hyperbolicity region of this model in the $\alpha_{2}=0$ plane. We see that already infinitely small values of the remaining coefficients are sufficient to enter the non-hyperbolic region and cause a loss of hyperbolicity. Additionally, the line $\alpha_{1}=-\alpha_{3}$ lies in the non-hyperbolic region.

\subsubsection{On the hyperbolicity loss of higher order models}

As the SWME cannot be written in closed form for the general case $N \in \mathbb{N}$ and a solution of the eigenvalue problem for large $N$ seems infeasible, a general analysis of the hyperbolicity regions proves difficult. However, the system can be reduced for a specific case that easily allows computation of the non-hyperbolic region also for higher order models.

We can extend Fig. 2 and numerically compute the hyperbolicity region of the corresponding moment models for large $N$ in the plane defined by $\alpha_{i}=0$ for $i=2, \cdots, N-1$, i.e. we only keep the first and the last coefficient $\alpha_{1}$ and $\alpha_{N}$. We see that the non-hyperbolic patches in this plane increase in number and size with increasing $N$. Furthermore, the equilibrium point $\alpha_{1}=\alpha_{N}=0$ is on the boundary of the hyperbolicity region, which means that the system can immediately become non-hyperbolic when starting from equilibrium. Also, we see that the line $\alpha_{1}=-\alpha_{N}$ always seems to be inside the non-hyperbolic region for small values of the coefficients.

However, it is not a-priori clear if distribution functions as considered non-hyperbolic in Fig. 2 ever occur during simulations. We want to investigate this by considering the non-hyperbolic values $\alpha_{1}=-\alpha_{N}, \alpha_{i}=0$ for $i=2, \cdots, N-1$, and use the scaling $\alpha_{N}=\widetilde{\alpha_{N}} \cdot u_{m}$, i.e. the following velocity distribution $u_{N}(t, x, \xi)$ for arbitrary $N \in \mathbb{N}$

$$
u_{N}(t, x, \xi)=u_{m}(t, x)\left(1+\widetilde{\alpha_{N}} \cdot\left(\Phi_{N}(\xi)-\Phi_{1}(\xi)\right)\right) .
$$

The boundary values are $u_{N}(t, x, 0)=1$ and $u_{N}(t, x, 1)=1+\left((-1)^{N}+1\right) \cdot \alpha$. In case of $\widetilde{\alpha_{N}}=1$, i.e. the coefficients are of the same order as the mean velocity, the function approximates 


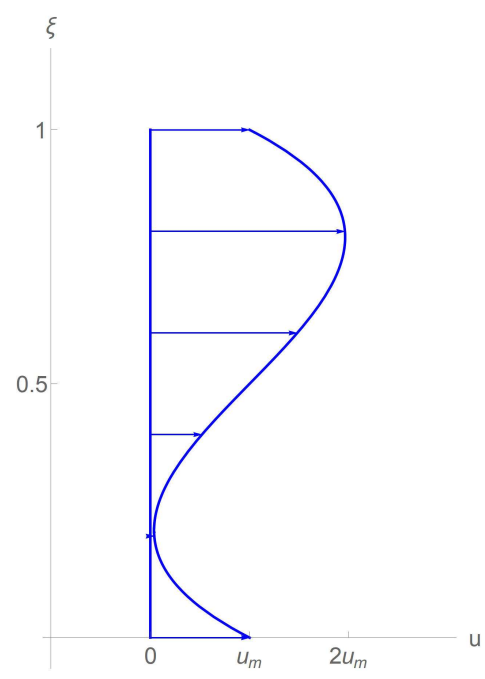

(a) $N=3$

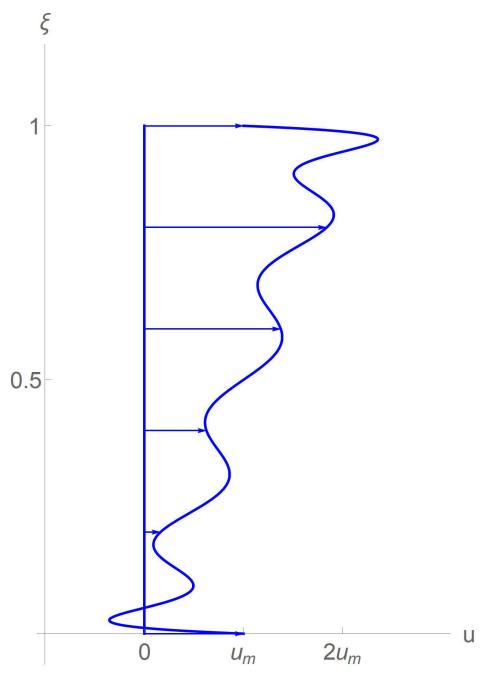

(b) $N=11$

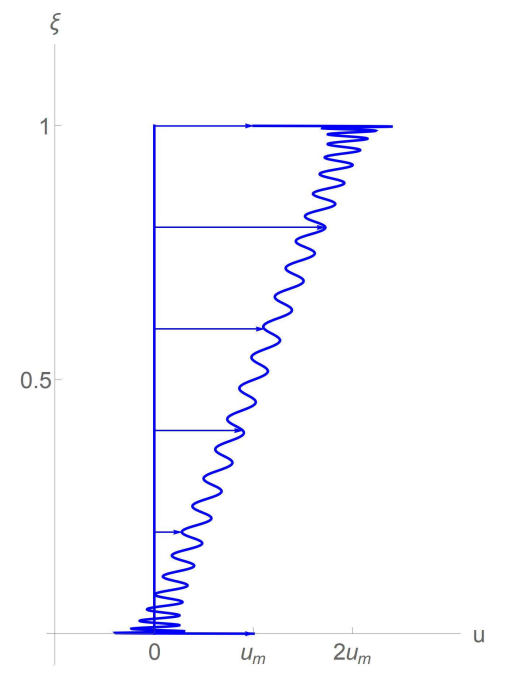

(c) $N=51$

Figure 3: Example velocity distributions for $N=3$ (left), $N=11$ (middle) and $N=51$ (right) that yield hyperbolicity loss.

the linear function $u(t, x, \xi)=2 u_{m} \xi$ that was commonly used in the test cases in [27], see also Fig. 3. We note that negativity of the distribution function is not necessary, as can be seen in the case $N=3$ and also for other values $\widetilde{\alpha_{N}}<1$. Therefore it seems evident that velocity distributions as mentioned above, can arise during simulation and the loss of hyperbolicity can occur.

In [27] the simulations for larger order $N \geq 2$ were performed using the eigenvalues for the $N=1$ model as an estimate, effectively avoiding the use of explicit and possibly complex eigenvalues of the higher order models in the numerical scheme. The maximum and minimum eigenvalues then reduce to the eigenvalues $\lambda_{1,2}$ from (2.11). However, this is only true for $\alpha_{i}=0, i \geq 2$, when

$$
\lambda_{1,2}=u_{m} \pm \sqrt{g h+\alpha_{1}^{2}} \quad \text { and } \quad \lambda_{3,4}=u_{m} \pm \sqrt{\frac{1}{5}} \alpha_{1} .
$$

For $\alpha_{i} \neq 0, i \geq 2$ the system matrix may be inconsistent with the eigenvalue estimates used in the numerical method.

For the smooth test cases in [27], the solution seems to run properly, but it might give nonphysical values upon loss of hyperbolicity as from that moment on the exact solution is no longer well defined. Reducing the eigenvalues to the first order system prevents any prediction of stability of the system, as there is an inconsistency between the used eigenvalues and the actual dynamics of the system. 


\subsection{Example of a breakdown of hyperbolicity}

In [27] the authors performed simulations of a smooth periodic wave interacting with its periodic reflection, which will be explained in more detail in Section 4 . In their simulation the system of higher order always used the (inconsistent) eigenvalues of the first order system. Following the previous section, we now give a simple example of physically plausible initial values that do lead to a loss of hyperbolicity at some times and in some points during the simulation even in the authors' simulation framework. We will show that the third order system is indeed not hyperbolic throughout the whole simulation.

The simulation setup closely follows [27], the initial values are $h(x)=1+$ $\frac{\exp (3 \cos (\pi(x+0.5)))}{\exp (4)}, u_{m}=0.25, \alpha_{1}=-0.25, \alpha_{2}=0$, and $\alpha_{3}=0.26$, which results in a cubic initial velocity profile

$$
u(0, x, \zeta)=-5.2 \zeta^{3}+7.8 \zeta^{2}-2.62 \zeta+0.26
$$

for all $x \in[-1,1]$ as depicted in Fig. 4 and marked in Fig. 2(b) by "•". This example is in fact very close to the values of hyperbolicity loss considered in Section 2.1.4, but still inside the hyperbolicity region. Plugging the initial values into the fifth order characteristic polynomial gives five real eigenvalues.

The velocity profile can occur during real-world applications, for example in situations where strong winds move the water surface or water is pumped into the channel by an industrial plant with a slightly higher velocity at the bottom.

Fig. 5 shows the evolution of the coefficients $\alpha_{1}, \alpha_{2}$ and $\alpha_{3}$ at three different spatial points $x_{1}=-0.5, x_{2}=0$ and $x_{3}=0.5$ during the simulation with green dots indicating hyperbolic states and red dots indicating loss of hyperbolicity. The single green dot in the top right of each graph shows the initial condition.

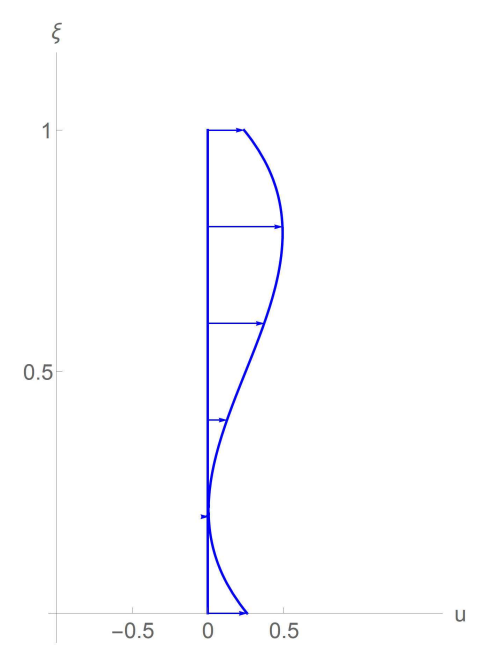

Figure 4: Cubic initial velocity profile close to hyperbolicity loss $u(0, x, \zeta)$ for $u_{m}=0.25$ and coefficients $\alpha_{1}=-0.25$, $\alpha_{2}=0$, and $\alpha_{3}=0.26$, Compare Fig. 3 . 


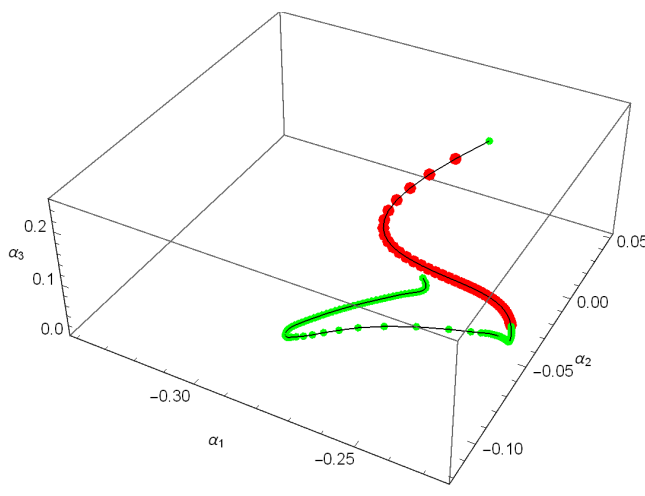

(a) $x_{1}=-0.5$

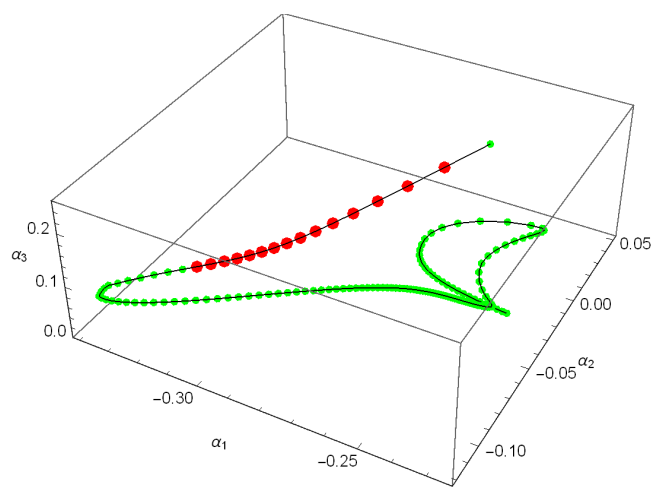

(b) $x_{2}=0$

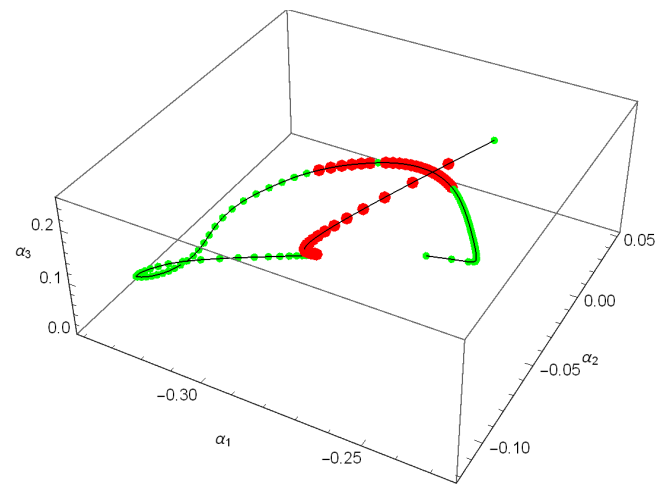

(c) $x_{3}=0.5$

Figure 5: SWME hyperbolic breakdown (red) at three different points in space (left $x_{1}=-0.5$; right $x_{2}=0$; bottom $x_{3}=0.5$ ) over time (see Fig. 4 and Table 1 for detailed setup).

Table 1: Simulation setup example of a breakdown of hyperbolicity test case.

\begin{tabular}{||c|l||}
\hline friction coefficient & $\lambda=0.1$ \\
\hline slip length & $v=0.1$ \\
\hline temporal domain & $t \in[0,2]$ \\
\hline spatial domain & periodic $x \in[-1,1]$ \\
\hline spatial resolution & $n_{x}=100$ \\
\hline initial height & $h(x)=1+\exp (3 \cos (\pi(x+0.5))) / \exp (4)$ \\
\hline initial velocity & $u(0, x, \zeta)=0.26-5.2 \zeta^{3}+7.8 \zeta^{2}-2.62 \zeta$ \\
\hline CFL number & $\approx 0.5$ \\
\hline numerical scheme & same as in [27] \\
\hline
\end{tabular}

It can clearly be seen that even though the simulation starts in a hyperbolic region, the simulation reaches non-hyperbolic states quickly. Several simulation steps at each plotted spatial point are thus using the wrong hyperbolic eigenvalues instead. It is unclear 


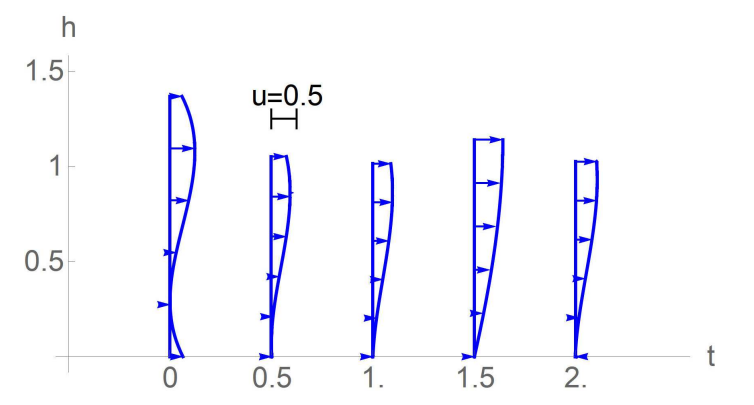

(a) $x_{1}=-0.5$

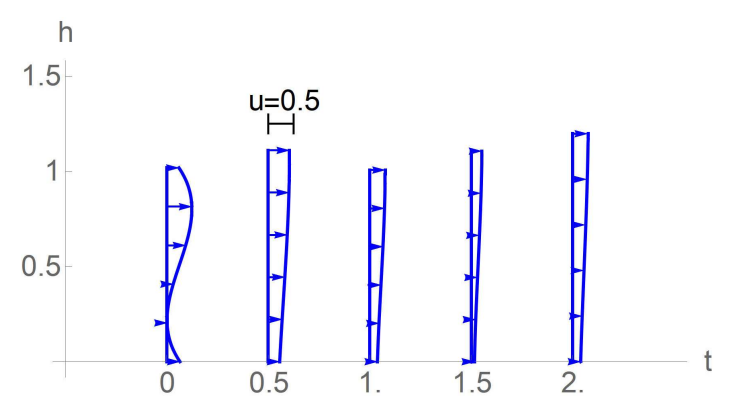

(b) $x_{2}=0$

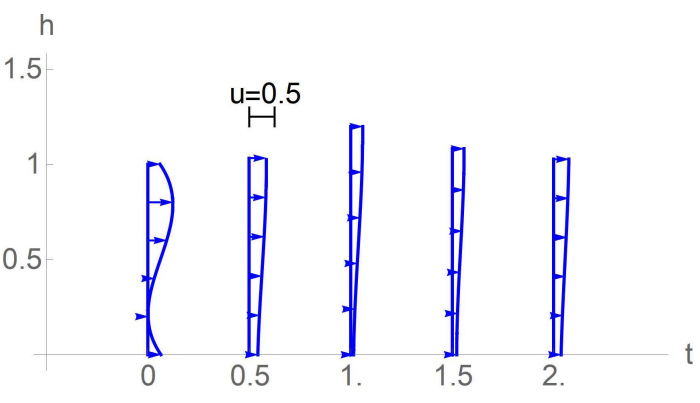

(c) $x_{3}=0.5$

Figure 6: Velocity profiles during hyperbolic breakdown test case at three different points in space (left $x_{1}=-0.5$; right $x_{2}=0$; bottom $x_{3}=0.5$ ) and different times (see Fig. 4 for detailed setup).

how accurate or meaningful the result of the simulation is as a proper Riemann solver for the underlying shallow water moment system would correctly break down due to complex eigenvalues. In Fig. 6 the corresponding velocity profiles at the three spatial points $x_{1}=-0.5, x_{2}=0$ and $x_{3}=0.5$ are plotted for different times. It can be seen that the velocity variation remains strongest in the region with initially large water height at $x=-0.5$, as the initial wave in this region results in faster water movement driving the non-equilibrium. The other points $x=0$ and $x=0.5$ relax to a uniform velocity distribution relatively quickly. However, the velocity distributions look reasonably physical at all times and positions and it is not obvious when and where the hyperbolicity loss occurs. The loss of hyperbolicity is therefore difficult to detect in the original simulation in [27].

During the simulations in [27] the system seemed to stay within hyperbolic regions for all times as claimed by the authors, but this can be attributed to the special choice of the initial condition. It is unclear how the loss of hyperbolicity can be prevented, especially given the non-linear dynamics of the system.

\subsection{Example of instability}

In [27] the numerical solution was performed using a third order WENO scheme for the conservative fluxes and a finite difference method for the non-conservative terms. 
Table 2: Simulation setup for example of instability test case.

\begin{tabular}{||c|l||}
\hline friction coefficient & $\lambda=0.1$ \\
\hline slip length & $v=0.01$ \\
\hline spatial domain & $x \in[-1,1]$ \\
\hline spatial resolution & $n_{x}=1000$ \\
\hline initial height & $h(x)= \begin{cases}5 & \text { if } x \leq 0, \\
1 & \text { if } x>0,\end{cases}$ \\
\hline initial velocity & $u(0, x, \zeta)=0.25-2.5 \zeta+7.5 \zeta^{2}-5 . \zeta^{3}$ \\
\hline CFL number & $\approx 0.7$ \\
\hline numerical scheme & UPRICE implementation $[21,36]$ \\
\hline
\end{tabular}

However, it is known that the treatment of the non-conservative terms requires special caution as different numerical schemes might yield different numerical solutions $[1,6$, 10]. This is especially true for the finite differences applied in [27] as this introduces a large amount of diffusion in comparison to the well-resolved conservative part. We therefore test the SHME equations again using a dedicated numerical method for the non-conservative terms. The implemented schemes are the same as in [22]. We use the path-consistent, first-order finite volume UPRICE scheme, described in [36]. The method was tested for comparable non-conservative models in rarefied gases in [21] and found to show well-resolved solutions, with especially little diffusion.

We perform the test case for $N=3$ and it is chosen such that the friction terms on the right hand are comparably small with respect to the convective terms, so that the coefficients only relax slowly to equilibrium. The parameters of the test case are given in Table 2. The initial condition is a dam break problem with initial velocity profile similar as in Fig. 4, which results in an initially non-hyperbolic cubic initial velocity profile.

In Fig. 7 the results are plotted for $t=0.05$ and $t=0.1$, together with a reference solution that has the same setup as in [27]. We see that the SWME model approximates the water height $h$ and velocity $u$ reasonably well. However, already the first coefficient $\alpha_{1}$ starts to show instabilities for this setup. The instabilities occur not at the leading shock wave, but behind that shockwave at an intermediate shock around $x=0.75$. This might be the reason why this could not be detected by the diffusive finite difference scheme in [27]. Any larger order moment system has several of those sub-shocks that might be the cause for instabilities in the system. It seems that the instabilities grow in time by comparison of the left and right figures.

In the rest of this paper, we want to circumvent the loss of hyperbolicity and the occurring instabilities by slightly changing the dynamics of the system resulting in a globally hyperbolic model that can use consistent eigenvalues without possible numerical inconsistencies. The regularized system will be represented by a modified system matrix $A_{H}$ having only real eigenvalues. 


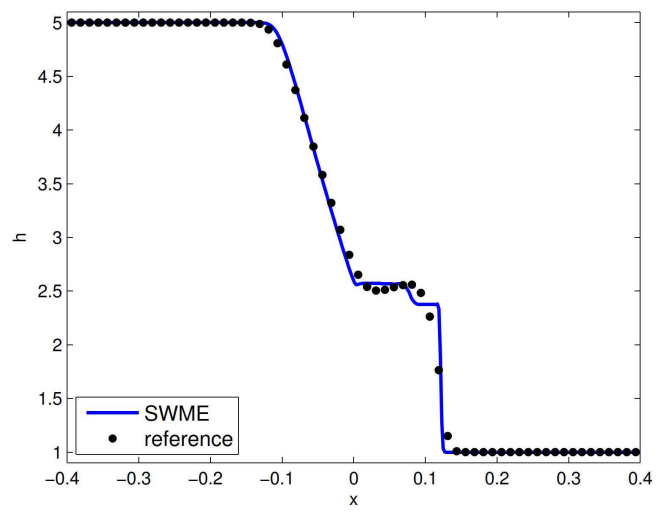

(a) $h, t=0.05$

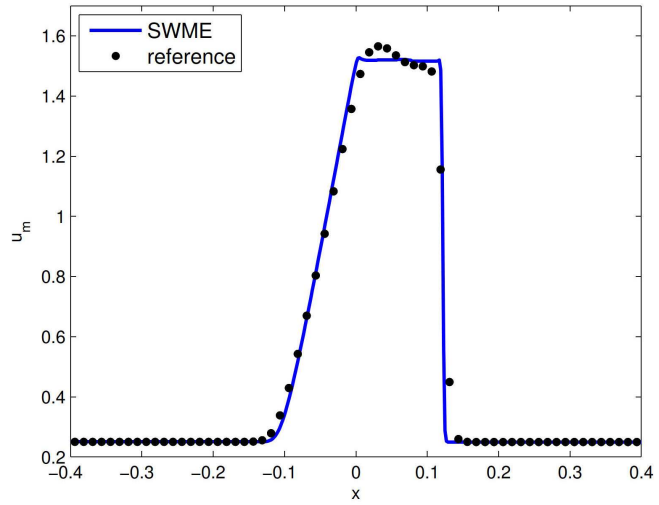

(c) $u_{m}, t=0.05$

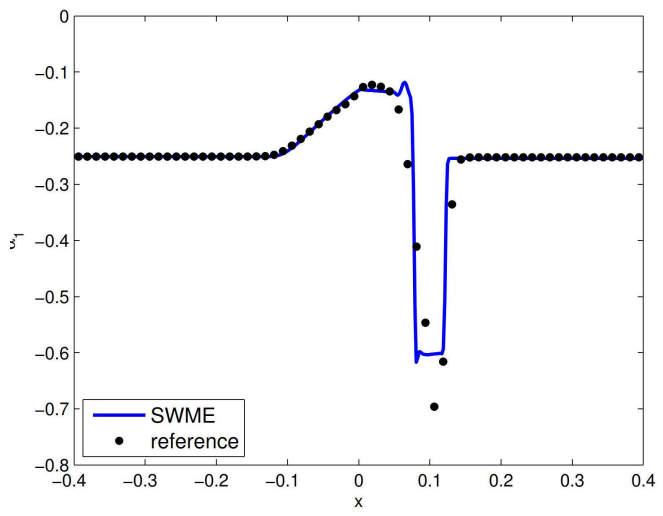

(e) $\alpha_{1}, t=0.05$

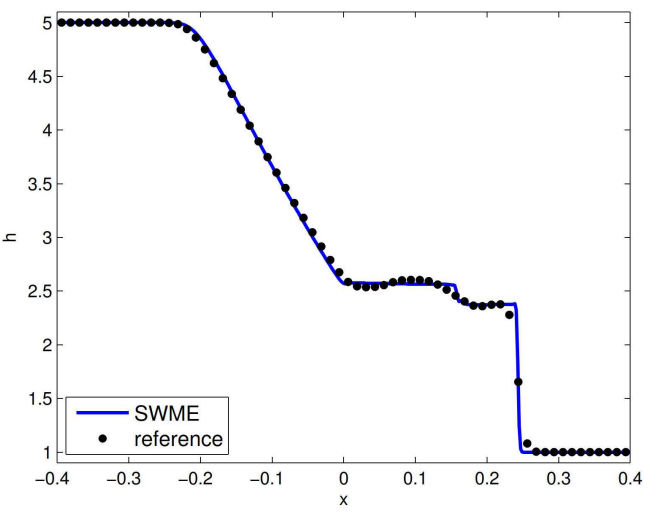

(b) $h, t=0.1$

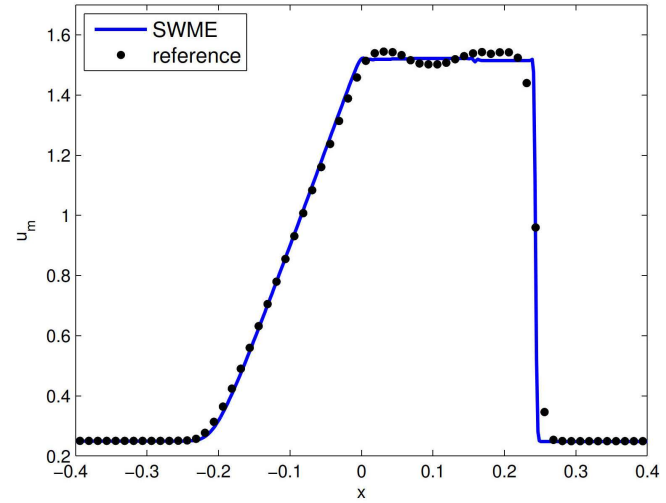

(d) $u_{m}, t=0.1$

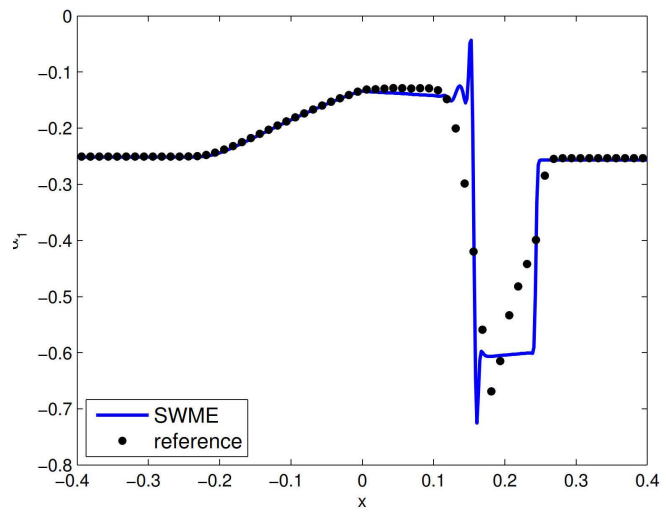

(f) $\alpha_{1}, t=0.1$

Figure 7: Unstable dam break simulation of SWME for $N=3$ at $t=0.05$ (left) and $t=0.1$ (right). Reference solution with settings from [27]. 


\section{Hyperbolic extension of shallow water moment equations}

The breakdown of hyperbolicity and instability in the previous section motivates the following sections to change the system matrix $A$ so that the system is hyperbolic.

In the first approach, all coefficients $\alpha_{i}$ of the system matrix, except the first one $\alpha_{1}$, are set to zero. This turns out to be very close to the corresponding system for the fixed eigenvalues (2.11) used in [27]. The resulting system is called Hyperbolic Shallow Water Moment Equations (HSWME) using a modified system matrix $A_{H}$.

The second approach is called generalized $\beta$-Hyperbolic Shallow Water Moment Equations ( $\beta$-HSWME). It is a generalization of the first approach achieving predefined propagation speeds. Hyperbolicity is guaranteed by introducing additional parameters $\beta_{i}$ in the last row of the system matrix. The corresponding system matrix is $A_{\beta}$.

\subsection{Hyperbolic Shallow Water Moment Equations (HSWME)}

We linearize the system matrix around the variables $\left(h, u_{m}, \alpha_{1}, 0, \cdots, 0\right)$, i.e.

$$
\left(h, u_{m}, \alpha_{1}, \cdots, \alpha_{N}\right) \rightarrow\left(h, u_{m}, \alpha_{1}, 0, \cdots, 0\right),
$$

or around linear deviations from equilibrium/constant velocity. Keeping $\alpha_{1}$ allows to capture a large part of the structure despite its simplicity. For example, there will still be a coupling between the different higher order equations.

We first derive the structure and the explicit form of the system matrix, before proving global hyperbolicity up to large $N$.

Theorem 3.1. The HSWME system matrix $A_{H} \in \mathbb{R}^{(N+2) \times(N+2)}$ is given by

$$
A_{H}=\left(\begin{array}{cccccc}
g h-u_{m}^{2}-\frac{1}{3} \alpha_{1}^{2} & 2 u_{m} & \frac{2}{3} \alpha_{1} & & & \\
-2 u_{m} \alpha_{1} & 2 \alpha_{1} & u_{m} & \frac{3}{5} \alpha_{1} & & \\
-\frac{2}{3} \alpha_{1}^{2} & & \frac{1}{3} \alpha_{1} & u_{m} & \ddots & \\
& & & \ddots & \ddots & \frac{N+1}{2 N+1} \alpha_{1} \\
& & & & \frac{N-1}{2 N-1} \alpha_{1} & u_{m}
\end{array}\right),
$$

where all other entries are zero.

Proof. See Appendix A.

Remark 3.1. In fact, our hyperbolic regularization strategy is similar as that in [2], where the system is linearized around equilibrium as well, but in conservative variables, then transformed to primitive variables. That way the system still looks highly non-linear, even though it is a linearization in another set of variables.

We can now prove the following property of the system matrix $A_{H}$. 
Theorem 3.2. The HSWME system matrix $A_{H} \in \mathbb{R}^{(N+2) \times(N+2)}$ (3.1) has the following characteristic polynomial

$$
\chi_{A}(\lambda)=\left(\left(\lambda-u_{m}\right)^{2}-g h-\alpha_{1}^{2}\right) \cdot \chi_{A_{2}}\left(\lambda-u_{m}\right),
$$

where the matrix $A_{2} \in \mathbb{R}^{N \times N}$ is defined as follows

$$
A_{2}=\left(\begin{array}{cccc} 
& c_{2} & & \\
a_{2} & & \ddots & \\
& \ddots & & c_{N} \\
& & a_{N} &
\end{array}\right),
$$

with values $c_{i}=\frac{i+1}{2 i+1} \alpha_{1}$ and $a_{i}=\frac{i-1}{2 i-1} \alpha_{1}$ the values above and below the diagonal, respectively, from (3.1).

Proof. See Appendix B.

With the help of the characteristic polynomial, we can now obtain the following result for the eigenvalues of HSWME.

Theorem 3.3. The eigenvalues of the system matrix $A_{H} \in \mathbb{R}^{(N+2) \times(N+2)}$ (3.1) are the real numbers

$$
\begin{aligned}
& \lambda_{1,2}=u_{m} \pm \sqrt{g h+\alpha_{1}^{2}}, \\
& \lambda_{i+2}=u_{m}+b_{i} \cdot \alpha_{1}, \quad i=1, \cdots, N,
\end{aligned}
$$

with $b_{i} \cdot \alpha_{1}$ the real and pairwise distinct roots of $A_{2}$ from Theorem 3.2 for all feasible orders of at least up to $N_{0}=150$.

The HSWME system is thus globally hyperbolic.

Proof. According to Theorem 3.2, the characteristic polynomial reads

$$
\chi_{A}(\lambda)=\left(\left(\lambda-u_{m}\right)^{2}-g h-\alpha_{1}^{2}\right) \cdot \chi_{A_{2}}\left(\lambda-u_{m}\right) .
$$

From the first factor, we immediately obtain

$$
\lambda_{1,2}=u_{m} \pm \sqrt{g h+\alpha_{1}^{2}} .
$$

Up to a shift with $u_{m}$ the last factor is the characteristic polynomial of the matrix

$$
A_{i}=\left(\begin{array}{cccc} 
& c_{i} & & \\
a_{i} & & \ddots & \\
& \ddots & & c_{N} \\
& & a_{N} &
\end{array}\right) \in \mathbb{R}^{N \times N},
$$


with $c_{i}=\frac{i+1}{2 i+1} \alpha_{1}$ and $a_{i}=\frac{i-1}{2 i-1} \alpha_{1}$.

Due to the structure of the matrix $A_{2}$, we can factor out $\alpha_{1}$ and see that the roots of the characteristic polynomial have the form $\lambda_{i}=u_{m}+b_{i} \cdot \alpha_{1}, i>2$.

This matrix is generating polynomials in $\lambda$ by the associated recursion formula

$$
\left|A_{i+2}\right|=\frac{1}{a_{i} c_{i}}\left(\left|A_{i}\right|+\widetilde{\lambda}\left|A_{i+1}\right|\right),
$$

similar to known sets of polynomials like Legendre or Hermite polynomials, which are generated by the same form of the so-called Jacobian matrix. We have computed the roots of $\left|A_{i}\right|$ up to large $i$ and only observed real roots up to $N_{0}=150$. A formal proof of this property seems to be difficult. However, the HSWME model will probably be used only for a small to medium number $N$, so that the numerical proof up to high order should be sufficient.

The HSWME model thus yields a globally hyperbolic system of equations for the simulation of shallow flows and can be seen both as an approximation to the original SWME and as an improvement due the lack of hyperbolicity of the SWME model.

Due to the real propagation speeds, the HSWME models do not loose hyperbolicity, unlike the SWME as shown in Section 2.2.

In the following we will show some examples of the HSWME model including the respective eigenvalues for higher order models.

\subsubsection{Example equations for HSWME}

For $N=0$ and $N=1$ the zeroth and first order system matrices of the HSWME are equivalent to the system matrices of the SWME for the zeroth and first order (2.1)-(2.2), (2.10), respectively.

For $N=2$ the second order system matrix of the HSWME reads

$$
A_{H}=\left(\begin{array}{cccc}
0 & 1 & 0 & 0 \\
g h-u_{m}^{2}-\frac{1}{3} \alpha_{1}^{2} & 2 u_{m} & \frac{2}{3} \alpha_{1} & 0 \\
-2 u_{m} \alpha_{1} & 2 \alpha_{1} & u_{m} & \frac{3}{5} \alpha_{1} \\
-\frac{2}{3} \alpha_{1}^{2} & 0 & \frac{1}{3} \alpha_{1} & u_{m}
\end{array}\right) .
$$

According to Theorem 3.3, we get the real propagation speeds

$$
\begin{aligned}
& \lambda_{1,2}=u_{m} \pm \sqrt{g h+\alpha_{1}^{2}}, \\
& \lambda_{3,4}=u_{m} \pm \sqrt{\frac{1}{5}} \alpha_{1} .
\end{aligned}
$$

These are in fact the proposed real eigenvalues (2.15) from [27]. 
Table 3: Eigenvalues of HSWME system matrix $A_{H}$ for order $N=0,1,2,3,4,5$.

\begin{tabular}{||c|c|c|c|c|c|c|c||}
\hline$N$ & EV 1 & EV 2 & EV 3 & EV 4 & EV 5 & EV 6 & EV7 \\
\hline 0 & $u_{m}+\sqrt{g h}$ & $u_{m}-\sqrt{g h}$ & - & - & - & - & - \\
\hline 1 & $u_{m}+\sqrt{g h+\alpha_{1}^{2}}$ & $u_{m}-\sqrt{g h+\alpha_{1}^{2}}$ & $u_{m}$ & - & - & - & - \\
\hline 2 & $u_{m}+\sqrt{g h+\alpha_{1}^{2}}$ & $u_{m}-\sqrt{g h+\alpha_{1}^{2}}$ & $u_{m}+\sqrt{\frac{1}{5}} \alpha_{1}$ & $u_{m}-\sqrt{\frac{1}{5}} \alpha_{1}$ & - & - & - \\
\hline 3 & $u_{m}+\sqrt{g h+\alpha_{1}^{2}}$ & $u_{m}-\sqrt{g h+\alpha_{1}^{2}}$ & $u_{m}+\sqrt{\frac{3}{7}} \alpha_{1}$ & $u_{m}-\sqrt{\frac{3}{7}} \alpha_{1}$ & $u_{m}$ & - & - \\
\hline 4 & $u_{m}+\sqrt{g h+\alpha_{1}^{2}}$ & $u_{m}-\sqrt{g h+\alpha_{1}^{2}}$ & $u_{m}+\sqrt{\left(\frac{1}{3}-\frac{2}{3 \sqrt{7}}\right)} \alpha_{1}$ & $u_{m}-\sqrt{\left(\frac{1}{3}-\frac{2}{3 \sqrt{7}}\right)} \alpha_{1}$ & $u_{m}+\sqrt{\left(\frac{1}{3}+\frac{2}{3 \sqrt{7}}\right)} \alpha_{1}$ & $u_{m}-\sqrt{\left(\frac{1}{3}+\frac{2}{3 \sqrt{7}}\right)} \alpha_{1}$ & - \\
\hline 5 & $u_{m}+\sqrt{g h+\alpha_{1}^{2}}$ & $u_{m}-\sqrt{g h+\alpha_{1}^{2}}$ & $u_{m}+\sqrt{\frac{15-2 \sqrt{15}}{33}} \alpha_{1}$ & $u_{m}-\sqrt{\frac{15-2 \sqrt{15}}{33}} \alpha_{1}$ & $u_{m}+\sqrt{\frac{15+2 \sqrt{15}}{33}} \alpha_{1}$ & $u_{m}-\sqrt{\frac{15+2 \sqrt{15}}{33}} \alpha_{1}$ & $u_{m}$ \\
\hline
\end{tabular}

For $N=3$ the third order system matrix of the HSWME is given by

$$
A_{H}=\left(\begin{array}{ccccc}
0 & 1 & 0 & 0 & 0 \\
g h-u_{m}^{2}-\frac{1}{3} \alpha_{1}^{2} & 2 u_{m} & \frac{2}{3} \alpha_{1} & 0 & 0 \\
-2 u_{m} \alpha_{1} & 2 \alpha_{1} & u_{m} & \frac{3}{5} \alpha_{1} & 0 \\
-\frac{2}{3} \alpha_{1}^{2} & 0 & \frac{1}{3} \alpha_{1} & u_{m} & \frac{4}{7} \alpha_{1} \\
0 & 0 & 0 & \frac{2}{5} \alpha_{1} & u_{m}
\end{array}\right)
$$

And again the propagation speeds from Theorem 3.3 coincide with the propagation speeds from [27]:

$$
\begin{aligned}
& \lambda_{1,2}=u_{m} \pm \sqrt{g h+\alpha_{1}^{2}}, \\
& \lambda_{3,4}=u_{m} \pm \sqrt{\frac{3}{7}} \alpha_{1}, \\
& \lambda_{5}=u_{m} .
\end{aligned}
$$

For $N=0, \cdots, 5$, Table 3 shows the eigenvalues of the corresponding modified matrices for larger order models. Interestingly, the eigenvalues EV 1 and EV 2 seem to be correct estimates for the maximum and minimum speeds in the system, which is a hint at the fact that the original numerical method used in [27] was not a bad choice. However, a full Riemann solver would still need to use the intermediate eigenvalues EV 3 - EV $(N+2)$.

The previous proofs give rise to a different regularization that allows for slightly more changes in the equations with the benefit of more flexibility with respect to the propagation speeds, which can be chosen almost arbitrarily. This approach will be discussed in the next section.

\section{$3.2 \beta$-Hyperbolic Shallow Water Moment Equations ( $\beta$-HSWME)}

We have seen that the HSWME propagation speeds are real even for a large number of equations in contrast to the SWME model. However, the eigenvalues $\lambda_{i}$ for $i>2$ are only obtained due to the simplifications of the model. They are bounded by the propagation 
speeds of the first order system but have no direct physical interpretation. In order to investigate the accuracy of the model in comparison to other possibly hyperbolic shallow water moment models, we want to derive other models having slightly different propagation speeds.

So we want to extend the HSWME approach towards more flexibility with respect to the propagation speeds and obtain a model with specific propagation speeds by allowing for small changes in the last equation of the system.

We note that even though the SWME are derived from first principles, it relies on a closure and is thus an approximation. The HSWME are indeed only an approximation of the physical process. We now want to see if it is possible to make more changes to the equations to obtain different properties and still achieve comparable accuracy. The numerical investigation then shows how accurate the model is. If the differences between the models are small, we could conclude that the approximation does not influence the accuracy. This would also mean that the moment approach in general is robust with respect to changes of the equations.

The main idea of the generalized $\beta$-HSWME for arbitrary $N$ is to add parameters $\beta_{i}$ so that the modified system matrix has predefined propagation speeds. Each parameter $\beta_{i}$ is added to the $i^{\text {th }}$ element of the last row of the modified matrix $A_{H}$ from Eq. (3.1). This way, the matrix is changed as little as possible only in the last row corresponding to the evolution equation for the last expansion coefficient, which should ideally be small in any case. Following Theorem 3.1, this defines

$$
A_{\beta}=\left(\begin{array}{cccccc}
g h-u_{m}^{2}-\frac{1}{3} \alpha_{1}^{2} & 2 u_{m} & \frac{2}{3} \alpha_{1} & & & \\
-2 u_{m} \alpha_{1} & 2 \alpha_{1} & u_{m} & \frac{3}{5} \alpha_{1} & & \\
-\frac{2}{3} \alpha_{1}^{2} & & \frac{1}{3} \alpha_{1} & u_{m} & \ddots & \\
& & & \ddots & \ddots & \frac{N+1}{2 N+1} \alpha_{1} \\
\beta_{1} & \ldots & & \beta_{N} & \beta_{N+1}+\frac{N-1}{2 N-1} \alpha_{1} & \beta_{N+2}+u_{m}
\end{array}\right) .
$$

The $\beta_{i}$ could have been added differently to the matrix. However, adding all $\beta_{i}$ to the last row does not further change the mass balance equation and all the other equations except the last. This means that mass conservation is still satisfied, while the momentum conservation is satisfied linearly around the linearization point. We will later give a very simple choice of all $\beta_{i}$ for which the matrix is indeed hyperbolic with additional Legendre eigenvalues.

Remark 3.2. Changing only the last entry of the system matrix is a well-known technique in moment models, where the last equation encodes the moment closure which is necessary because the moment expansion in Eq. (2.5) can in general be infinite. This leads to an infinite and coupled system that has to be closed somehow. See e.g. $[2,31,38]$ for different moment closures. This could also include changes such that the system has other desirable properties like hyperbolicity, or conservation of higher order moments. 


\subsubsection{Hyperbolicity proof for arbitrary but distinct eigenvalues}

In this subsection it will be shown that there exists a unique solution for the parameters $\beta_{i}$ for any given set of $N+2$ distinct eigenvalues of the matrix $A_{\beta}$.

Lemma 3.1. Let $A_{\beta} \in \mathbb{R}^{(N+2) \times(N+2)}$ from (3.4) with $N \geq 2$,

$$
\chi\left(\lambda, \beta_{1}, \cdots, \beta_{N+2}\right)
$$

the characteristic polynomial of $A_{\beta}$ and

$$
S\left(\lambda, b_{1}, \cdots, b_{N+2}\right)=(-1)^{N+2}\left(\lambda-b_{1}\right)\left(\lambda-b_{2}\right) \cdots\left(\lambda-b_{N+2}\right)
$$

a polynomial with roots $b_{i}, i=1, \cdots, N+2$.

Then there exists a unique solution for the parameters $\beta_{i}^{S}$ so that the characteristic polynomial matches the target polynomial, i.e.

$$
\chi\left(\lambda, \beta_{1}^{S}, \cdots, \beta_{N+2}^{S}\right)=S\left(\lambda, b_{1}, \cdots, b_{N+2}\right) .
$$

Proof. $A_{\beta}$ only has one non-zero upper diagonal. We compute the characteristic polynomial expanding the determinant with respect to the last column.

$$
\begin{aligned}
\chi\left(\lambda, \beta_{1}, \cdots, \beta_{N+2}\right) & =\operatorname{det}\left(A_{\beta}-\lambda I\right) \\
& =-\frac{N+1}{2 N+1} \alpha_{1} \operatorname{det}\left(M_{N+1, N+2}\right)+\left(\beta_{N+2}+u_{m}-\lambda\right) \operatorname{det}\left(M_{N+2, N+2}\right),
\end{aligned}
$$

with $M_{i, j}$ being the corresponding minors. Continuing to expand the determinants along the last column, one can easily see with (3.4) that the characteristic polynomial of the matrix $A_{\beta}$ will be of the form

$$
\begin{aligned}
\chi\left(\lambda, \beta_{1}, \cdots, \beta_{N+2}\right)= & (-1)^{N+2} \lambda^{N+2}+a_{N+2}\left(\beta_{N+2}\right) \lambda^{N+1} \\
& +a_{N+1}\left(\beta_{N+2}, \beta_{N+1}\right) \lambda^{N}+\cdots+a_{0}\left(\beta_{N+2}, \cdots, \beta_{1}\right),
\end{aligned}
$$

where the coefficients $a_{j}$ depend only linearly on the parameters $\beta_{i}$ with $i \geq j$, since every parameter appears in the matrix only once. Following the fundamental theorem of algebra, the polynomial $\chi\left(\lambda, \beta_{N+2}, \cdots, \beta_{1}\right)$ can be expressed by its roots in $\mathbb{C}$. Now we set

$$
\chi\left(\lambda, \beta_{1}, \cdots, \beta_{N+2}\right)=S\left(\lambda, b_{1}, \cdots, b_{N+2}\right) .
$$

Since the coefficients $a_{j}$ depend only linearly on $\beta_{i}$, the equation can be solved uniquely by matching coefficients resulting in the values for $\beta_{i}^{S}$.

Remark 3.3. Note that the previous proof only works if all $\beta_{i}$ are added to different columns, otherwise there would be multiplicative terms in $\beta_{i}$ so that the unique solution might not exist. 
We use the previous Lemma 3.1 to show the hyperbolicity of $A_{\beta}$.

Theorem 3.4. There always exists a set of parameters $\beta_{1}^{S}, \cdots, \beta_{N+2}^{S}$ so that the system

$$
\frac{\partial V}{\partial t}+A_{\beta} \frac{\partial V}{\partial x}=P
$$

is globally hyperbolic with $\beta_{i}=\beta_{i}^{S}$ for $N \geq 2$ and the eigenvalues match the given pairwise distinct real roots $b_{i}$, for $i=1, \cdots, N+2$.

Proof. Choosing the target roots $b_{i}$ before setting the target characteristic polynomial as $S(\lambda)=(-1)^{N+2}\left(\lambda-b_{1}\right)\left(\lambda-b_{2}\right) \cdots\left(\lambda-b_{N+2}\right)$, and using Lemma 3.1 by setting

$$
\chi\left(\lambda, \beta_{1}, \cdots, \beta_{N+2}\right)=S(\lambda)
$$

we get a unique solution $\beta_{i}^{S}$ for each set of parameters $b_{i}$. The system matrix $A_{\beta}$ with $\beta_{i}=\beta_{i}^{S}$ has real eigenvalues $b_{1}, \cdots, b_{N+2}$. Since all eigenvalues are distinct, the system is globally hyperbolic.

\subsubsection{System matrix $A_{\beta}$ of $\beta$-HSWME}

The proof shown above is a constructive proof. Therefore the following algorithm can be performed to obtain the hyperbolic system matrix of the $\beta$-HSWME with the propagation speeds $b_{1} \cdots b_{N+2}$ :

1. Add parameters $\beta_{i}$ to the last row of the system matrix of HSWME to obtain the matrix $A_{\beta}$ and calculate the characteristic polynomial $\chi\left(\lambda, \beta_{1}, \cdots, \beta_{N+2}\right)$.

2. Choose target propagation speeds $b_{i}$ that should be the desired eigenvalues of the system matrix. See Remark 3.5 for more details.

3. Solve for the uniquely defined $\beta_{i}$ by matching coefficients of the target polynomial $S(\lambda)=(-1)^{N+2}\left(\lambda-b_{1}\right) \cdots\left(\lambda-b_{N+2}\right)$ and the characteristic polynomial $\chi\left(\lambda, \beta_{1}, \cdots, \beta_{N+2}\right)$.

4. Insert the solution $\beta_{i}$ to obtain the explicit $A_{\beta}$.

\subsubsection{Example for $\beta$-HSWME using Legendre eigenvalues}

For an example of the $\beta$-HSWME model for $\geq 2$ we follow the steps from the previous section and choose shifted and scaled Legendre roots as target propagation speeds. 
1. Adding parameters $\beta_{i}$ to the last row of $A_{H}$ leads to $A_{\beta}$

$$
A_{\beta}=\left(\begin{array}{cccccc}
g h-u_{m}^{2}-\frac{1}{3} \alpha_{1}^{2} & 2 u_{m} & \frac{2}{3} \alpha_{1} & & & \\
-2 u_{m} \alpha_{1} & 2 \alpha_{1} & u_{m} & \frac{3}{5} \alpha_{1} & & \\
-\frac{2}{3} \alpha_{1}^{2} & & \frac{1}{3} \alpha_{1} & u_{m} & \ddots & \\
& & & \ddots & \ddots & \frac{N+1}{2 N+1} \alpha_{1} \\
\beta_{1} & \ldots & & \beta_{N} & \beta_{N+1}+\frac{N-1}{2 N-1} \alpha_{1} & \beta_{n+2}+u_{m}
\end{array}\right)
$$

and the characteristic polynomial $\chi\left(\lambda, \beta_{1}, \cdots, \beta_{N+2}\right)$ can be computed using some numerical algebra software.

2. For the desired eigenvalues $b_{i}$ of the system, we use the following example:

$$
\begin{aligned}
& b_{1}=u_{m}-\sqrt{g h+\alpha_{1}^{2}}, \\
& b_{2}=u_{m}+\sqrt{g h+\alpha_{1}^{2}}, \\
& b_{i+2}=u_{m}+c_{i, N} \alpha_{1}, \quad i=1, \cdots, N,
\end{aligned}
$$

where $c_{i, N}$ is the $i$-th root of the Legendre polynomial of degree $N$, symmetrically placed within the interval $[-1,1]$. This means that all eigenvalues $b_{i}$ are real. Furthermore, all eigenvalues are within the interval $\left[u_{m}-\sqrt{g h+\alpha_{1}^{2}}, u_{m}+\sqrt{g h+\alpha_{1}^{2}}\right]$, which corresponds to the propagation speeds of the first order SWME.

3. Matching coefficients of the characteristic polynomial $\chi\left(\lambda, \beta_{1}, \cdots, \beta_{N+2}\right)$ and the target polynomial $S(\lambda)=(-1)^{N+2}\left(\lambda-b_{1}\right) \cdots\left(\lambda-b_{N+2}\right)$, we can compute the unique solution of values $\beta_{i}$ according to Theorem 3.4. For this example, the simple solution reads

$$
\begin{aligned}
& \beta_{i}=0, \quad i \neq N+1, \\
& \beta_{N+1}=\frac{N^{2}-N}{2 N^{2}+N-1} \alpha_{1} .
\end{aligned}
$$

The regularization entry $\beta_{N+1}$ is monotonically increasing and bounded by the limit

$$
\lim _{N \rightarrow \infty} \beta_{N+1}=\frac{1}{2}
$$

leading to a small change in the last equation of the system even for large $N$. 
4. The new system matrix reads

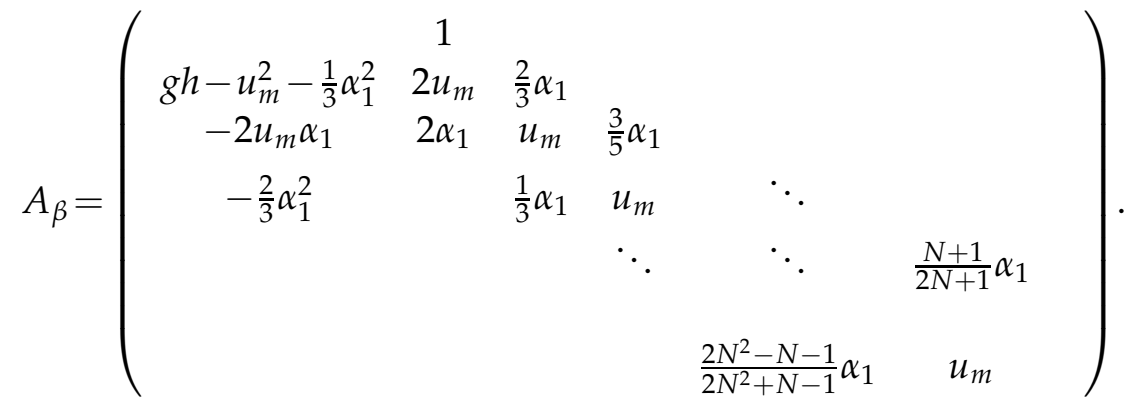

The modified entry in the last row is bounded by 1 .

We summarize the result of the example above in the following theorem.

Theorem 3.5. The $\beta$-HSWME model with only non-zero parameter

$$
\beta_{N+1}=\frac{N^{2}-N}{2 N^{2}+N-1} \alpha_{1}
$$

is hyperbolic with eigenvalues

$$
\begin{aligned}
& \lambda_{1,2}=u_{m} \pm \sqrt{g h+\alpha_{1}^{2}}, \\
& \lambda_{i+2}=u_{m}+c_{i, N} \cdot \alpha_{1}, \quad i=1, \cdots, N,
\end{aligned}
$$

where $c_{i, N}$ is $i-t$ th root of the Legendre polynomial of degree $N$ on $[-1,1]$ for all feasible orders of at least up to $N_{0}=100$.

Proof. Analogously to Theorem 3.3, we directly get the first two eigenvalues and the remaining eigenvalues can be obtained with the help of the submatrix

$$
A_{2}^{\beta}=\left(\begin{array}{cccc} 
& c_{2} & & \\
a_{2} & & \ddots & \\
& \ddots & & c_{N} \\
& & \beta_{N+1}+a_{N} &
\end{array}\right),
$$

with $\beta_{N+1}=\frac{N^{2}-N}{2 N^{2}+N-1} \alpha_{1}$.

By numerical computation up to large order (we have conducted numerical tests up to order $N_{0}=100$ ), we show that the characteristic polynomial is the same as the Legendre polynomial of order $N$. The remaining roots of $A_{\beta}$ are thus given according to the Legendre roots $c_{i, N}$ of order $N$ by $u_{m}+c_{i, N} \cdot \alpha_{1}$. 
Remark 3.4. For $N=1$, SWME, HSWME and $\beta$-HSWME are equivalent.

For $N=2$, the $\beta$-HSWME model uses two non-zero parameters given by

$$
\beta_{1}=-\frac{4}{9} \alpha_{1}^{2}, \quad \beta_{3}=\frac{2}{9} \alpha_{1},
$$

and the hyperbolicity proof including the eigenvalues of the system can be easily done by direct computation of the eigenvalues.

Table 4 summarizes the model hierarchy including the maximal order $N$ so that the corresponding system is hyperbolic and the respective structure of the propagation speeds. The standard SWE correspond to $N=0$. The SWME derived as an extension of the shallow water equations are hyperbolic up to order $N=1$ and do not yield an analytical formula for the eigenvalues. The new HSWME are hyperbolic for all tested $N \in \mathbb{N}$, with $N \leq 150$ and the structure of the propagation speed can be computed. Based on that, the new $\beta$-HSWME is hyperbolic for all $N \in \mathbb{N}$ and yields almost arbitrary propagation speeds, with one example illustrated in this section.

Table 4: Model hierarchy overview including hyperbolicity and eigenvalue structure.

\begin{tabular}{||c|c|c||}
\hline model & hyperbolic for & propagation speeds \\
\hline SWE & $N=0$ & $\lambda_{1,2}=u_{m} \pm \sqrt{g h}$ \\
\hline SWME & only $N \leq 1$ & not analytical \\
\hline HSWME & at least $N \leq 150$ & $\begin{array}{c}\lambda_{1,2}=u_{m} \pm \sqrt{g h+\alpha_{1}^{2}} \\
\lambda_{i+2}=u_{m}+b_{i} \cdot \alpha_{1} \quad \text { for fixed } b_{i} \in \mathbb{R}\end{array}$ \\
\hline$\beta$-HSWME & $N \in \mathbb{N}$ & arbitrary, e.g. $\begin{array}{c}\lambda_{1,2}=u_{m} \pm \sqrt{g h+\alpha_{1}^{2}} \\
\lambda_{i+2}=u_{m}+c_{i, N} \cdot \alpha_{1} \quad \text { for } c_{i, N} \in \mathbb{R}\end{array}$ \\
\hline
\end{tabular}

Remark 3.5. In this paper, we focus on one choice for the propagation speeds for the $\beta$-HSWME model. However, different choices are possible. In the given realisation of the $\beta$-HSWME model, the propagation speeds are chosen in accordance to the following points:

1. The model should include the underlying shallow water model in equilibrium, i.e. $u_{m} \pm \sqrt{g h}$ should be obtained for vanishing coefficients

2. The model should be invariant with respect to changes in $u_{m}$, i.e. $u_{m}$ is an additive term in all eigenvalues.

3. The remaining part of the propagation speeds should depend on $\alpha_{1}$.

4. The model should reflect the choice of the basis functions, which are Legendre functions in this case. 
Points 1 and 2 are obvious from the underlying SWE. Point 3 seems reasonable as the underlying HSWME model contains only $\alpha_{1}$ as higher order variable in the matrix and especially the lower rows of the matrix. Point 4 is chosen to resemble similar moment models from rarefied gases, where the discretization using Hermite polynomials leads to shifted and scaled Hermite roots as propagation speeds [22]. Different choices are possible, especially in other applications of moment models, other underlying models or discretizations, but this is beyond the scope of this paper.

\subsection{Physical interpretation of hyperbolic regularizations}

Both the HSWME and the $\beta$-HSWME model succeed in overcoming possible stability problems of the SWME model by guaranteeing that the eigenvalues of the new models are always real. However, additional changes might impact the physical properties of the model. We want to emphasize that both the HSWME and the $\beta$-HSWME model do not modify the two most important propagation speeds $u_{m} \pm \sqrt{g h}$, which belong to the underlying shallow water equations. The conservation of mass is not altered and the momentum equation is simplified by removing the some nonlinear terms corresponding to the higher order coefficients $\alpha_{i}$ for $i>1$. This leads to less back coupling of the higher order coefficients/equations to the lower order equations for mass and momentum, and similarly for the other equations in between. In numerical computations of moment equations, we can expect a structure of subshocks that reflects the hyperbolic nature of the system. The new, hyperbolic models use less term for the back coupling of the higher order coefficients to the lower order coefficients, especially in relation to the friction terms on the right hand side, which remain unchanged. It thus seems reasonable that the subshocks for the HSWME and $\beta$-HSWME model are reduced. In other words, the subshocks in higher order coefficients do not influence the lower order coefficients as much as in the SWME model. In cases, where the higher order coefficients are different from zero, the HSWME and $\beta$-HSWME models are thus expected to give smoother results. One example is the test case, where the SWME model suffered instabilities in Fig. 7.

The model is still highly nonlinear. The differences of SWME and the new models are only in the nonlinear terms of higher order. Those terms small in case of small deviation from linear velocity profiles. Despite the reduced back coupling, the effect of $\alpha_{1}$ on the other equations remains largely unchanged. In cases of linear velocity profiles or small deviations thereof, the introduced approximation is thus believed to result in small errors from the original model. In cases, where the velocity profile is deviating significantly from a linear (or constant) profile, for example in cases of very large $N$ and large values of the higher order coefficients, the approximation quality will obviously deteriorate. We note that this represents a difficult test case anyway, as large high order coefficients correspond to highly non uniform velocity profiles. However, for the typical use case of small $N$ and reasonably complex (i.e. linear or small deviations) velocity profiles, the new HSWME and $\beta$-HSWME models might give satisfactory results with guaranteed 
hyperbolic equations.

The additional term in the last row of the $\beta$-HSWME model leads to an increased influence of $\alpha_{N-1}$ on the evolution of the last coefficient $\alpha_{N}$. This also means that information propagates faster from the lower order equations to the last equation. In order to satisfy the last balance law equation, it is thus not necessary for the last coefficient $\alpha_{N}$ to show too much variation. By means of the limited back coupling, this feeds back into the equation for $\alpha_{N-1}$ and is expected to smooth the equation there.

We will come back to these interpretations when looking at the results of the HSWME and the $\beta$-HSWME model in comparison with the SWME model in Section 4.

\subsection{Linear Stability Analysis}

In this section the SWME, the HSWME and the $\beta$-HSWME with the Legendre target polynomial from the example of the previous Section 3.2.3 shall be analyzed with respect to their linear stability. Linear stability is a crucial property of moment models, as also investigated in [11]. We assume a small perturbation $\delta=V_{\text {pert }}-V_{0}$ around a linearization point $V_{0}$ and consider the linearized system

$$
\frac{\partial \delta}{\partial t}+A\left(V_{0}\right) \frac{\partial \delta}{\partial x}=\frac{\partial P\left(V_{0}\right)}{\partial V} \delta .
$$

The right hand side of the linearized system is obtained from the right side of Eq. (2.9) using the linearization

$$
P\left(V_{\text {pert }}\right)=P\left(V_{0}\right)+\frac{\partial P\left(V_{0}\right)}{\partial V}\left(V_{\text {pert }}-V_{0}\right)+\mathcal{O}\left(\left(V_{\text {pert }}-V_{0}\right)^{2}\right) \approx P\left(V_{0}\right)+\frac{\partial P\left(V_{0}\right)}{\partial V} \delta,
$$

so that $P(\delta)=P\left(V_{\text {pert }}-V_{0}\right) \approx \frac{\partial P\left(V_{0}\right)}{\partial V} \delta$. As system matrices $A\left(V_{0}\right)$ we use the matrices $A$, $A_{H}$, and $A_{\beta}$ from (2.12), (3.3) and (3.5) evaluated at $V_{0}$.

For the perturbation we use a wave ansatz

$$
\delta=c e^{i(k x-\omega t)}=c e^{i k x} e^{-i \omega t},
$$

where $c \in \mathbb{R}^{N+2}$ is the amplitude of the wave, $k \in \mathbb{R}$ is the wave number and $\omega \in \mathbb{C}$ is the complex frequency. Since $\delta$ represents the perturbation from the perturbation point $V_{0}$, the imaginary part of the frequency $\operatorname{Im}(\omega)$ should not be positive for stability in time, i.e.

$$
\operatorname{Im}(\omega) \leq 0 .
$$

Plugging the perturbation into Eq. (3.6) and rearranging gives

$$
\begin{aligned}
0 & =-i \omega \delta+i k A\left(V_{0}\right) \delta-\frac{\partial P\left(V_{0}\right)}{\partial V} \delta \\
& =\left(-i \omega I+i k A\left(V_{0}\right)+\frac{\partial P\left(V_{0}\right)}{\partial V}\right) \delta .
\end{aligned}
$$


Table 5: Fixed values of the ground state $V_{0}$ for linear stability analysis.

\begin{tabular}{||c|c|c|c|c|c|c|c||}
\hline$h$ & $u_{m}$ & $\alpha_{1}$ & $\alpha_{2}$ & $g$ & $v$ & $\lambda$ & $\mathrm{k}$ \\
\hline 1 & 0.25 & 0 & 0 & 1 & 0.1 & 0.1 & 1 \\
\hline
\end{tabular}

By multiplying with $-i$ we get

$$
0=\left(\omega I-k A\left(V_{0}\right)+i \frac{\partial P\left(V_{0}\right)}{\partial V}\right) \delta .
$$

So that $\omega$ needs to be an eigenvalue of $-k A\left(V_{0}\right)+i \frac{\partial P\left(V_{0}\right)}{\partial V}$ for non-trivial solutions.

Investigating the eigenvalues of this matrix, we check if the condition (3.7) for $\omega$ is fulfilled. We focus on the case $N=2$, the other systems can be treated in the same way yielding similar results.

We choose the values of $V_{0}$ and additional coefficients as presented in Table 5 according to the values in the simulation of [27].

We then use $\alpha_{1}$ and $\alpha_{2}$ as perturbations from the linearization point and analyze the eigenvalues of the system.

In Fig. 8, the beige regions show the stable regions, while the blue regions show the unstable regions.

We see that the SWME, HSWME, and $\beta$-HSWME are all linearly stable for small values of $\alpha_{1}$ and $\alpha_{2}$.

\section{Numerical simulation}

We first demonstrate that the HSWME and $\beta$-HSWME models overcome the stability problems of the SWME model shown in Fig. 7 and then test the HSWME and $\beta$-HSWME models numerically using the smooth test case from [27] and another non smooth dam break test case.

We choose the moment order up to $N=4$ and compare it with the original SWME system and a reference solution from [27]. The numerical simulations are performed using the path-conservative numerical UPRICE method given in [36] as explained before. We emphasize that a direct Roe solver, for example as described within [22], does break down for SWME in case of hyperbolicity loss. We note that the new models HSWME and $\beta$-HSWME are significantly easier to implement and to solve, as the hyperbolic regularization reduces the number of non linear terms drastically for the larger values of $N$. The $\beta$-HSWME has effectively the same runtime as the HSWME model.

\subsection{Overcoming instability of SWME}

First we repeat the test case from Section 2.3, where the SWME showed instabilities in the first coefficient $\alpha_{1}$. The simulations for HSWME and $\beta$-HSWME use the same settings as for SWME given in Table 1. The results are shown in Fig. 9. 


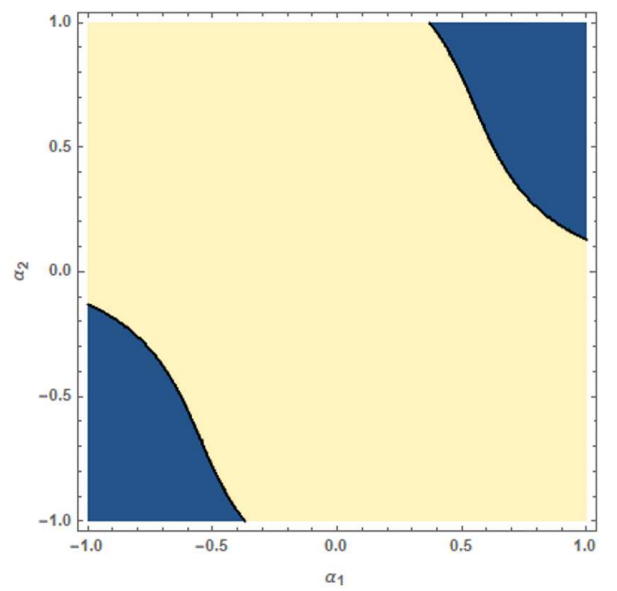

(a) SWME

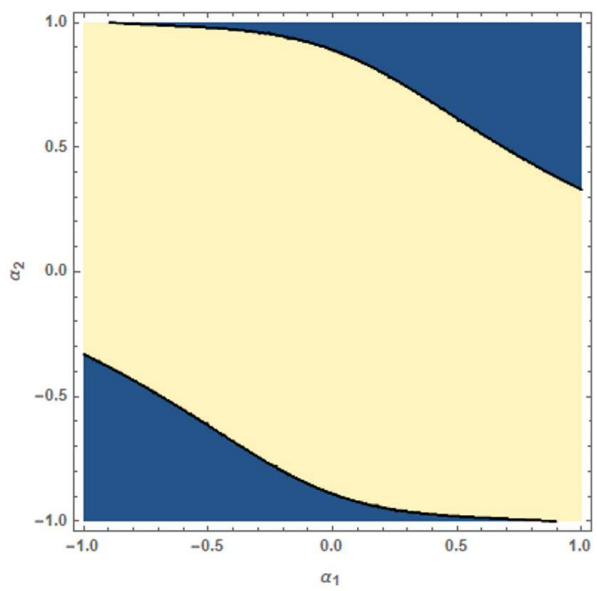

(b) HSWME

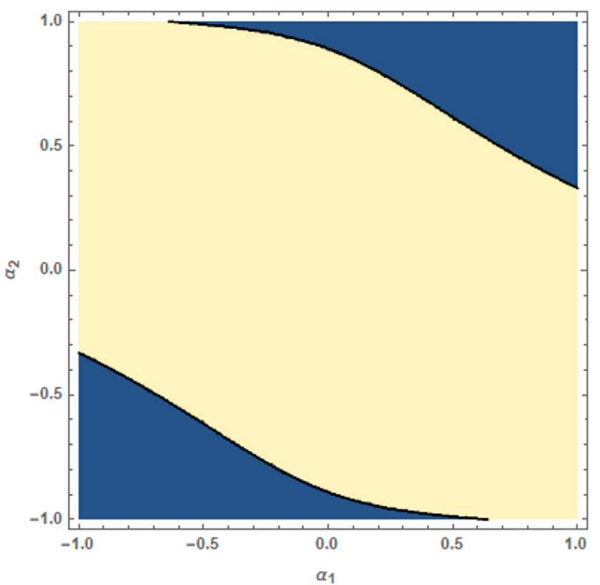

(c) $\beta$-HSWME

Figure 8: Linearly stable (beige) and unstable (blue) regions for SWME (top left), HSWME (top right) and $\beta$-HSWME (bottom) depending on $\alpha_{1}$ and $\alpha_{2}$ (see Table 5).

The results for the water height $h$ in Figs. 9(a) and 9(b) show only small differences for the new hyperbolic models. However, both models (on top of each other) have a smoother behavior at the plateau for $x \in[0,0.12]$ for $t=0.05$ and for $x \in[0,0.25]$ for $t=0.05$, respectively. This coincides with the interpretation in Section 3.3. The changing values in the higher order variables apparently do not influence the water height as much as in the SWME model due to the reduced back coupling. This can be interpreted as a regularizing effect of the model, as the additional sub shock in the SWME model can be unphysical and lead to the oscillations visible for $\alpha_{1}$. The mean velocity $u_{m}$ is visually the same for all models. For the first coefficient in Figs. 9(e) and 9(f) we now see the same regularizing effect mentioned earlier. The oscillations of the SWME model are greatly reduced if 


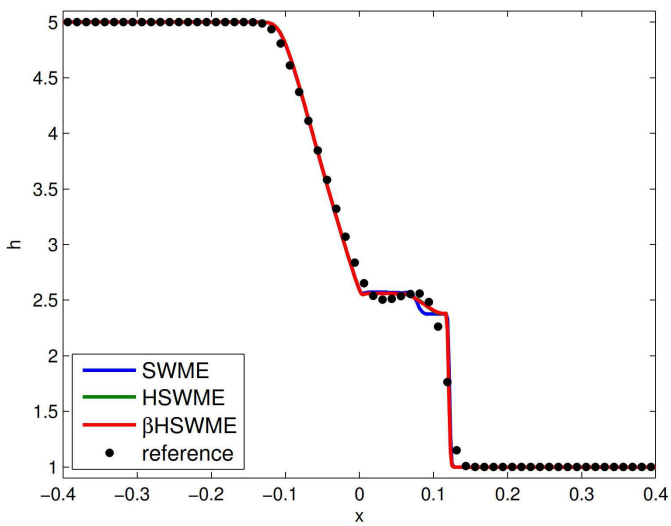

(a) $h, t=0.05$

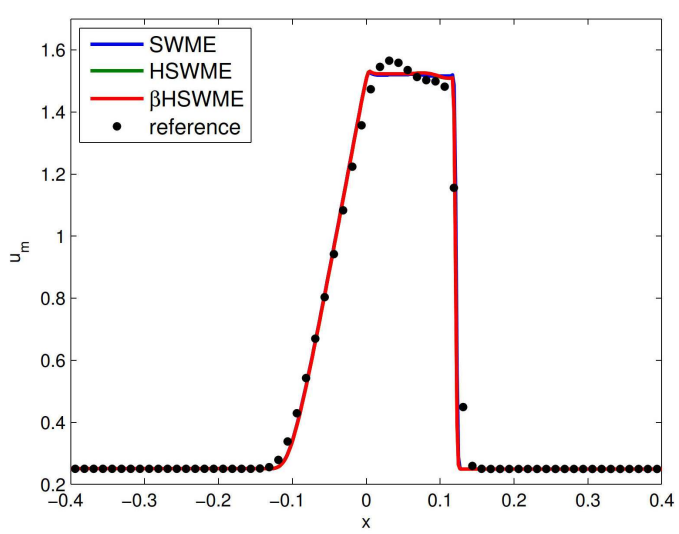

(c) $u_{m}, t=0.05$

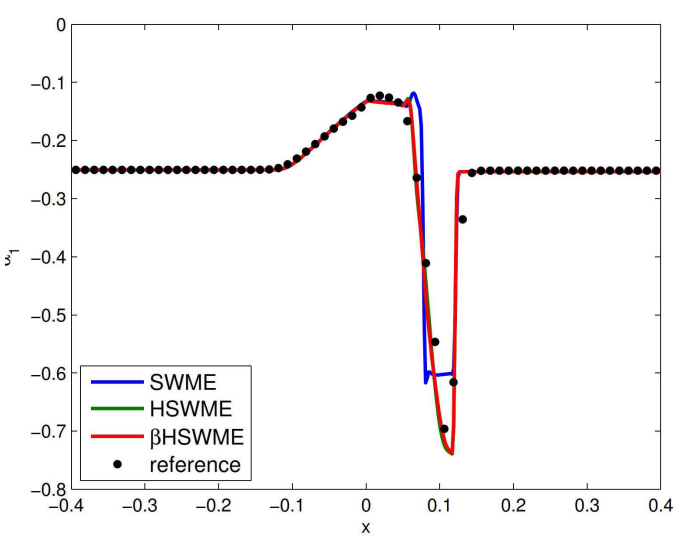

(e) $\alpha_{1}, t=0.05$

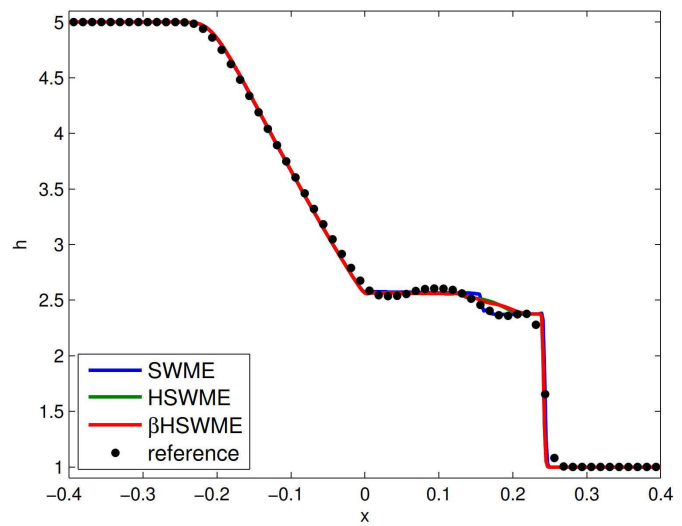

(b) $h, t=0.1$

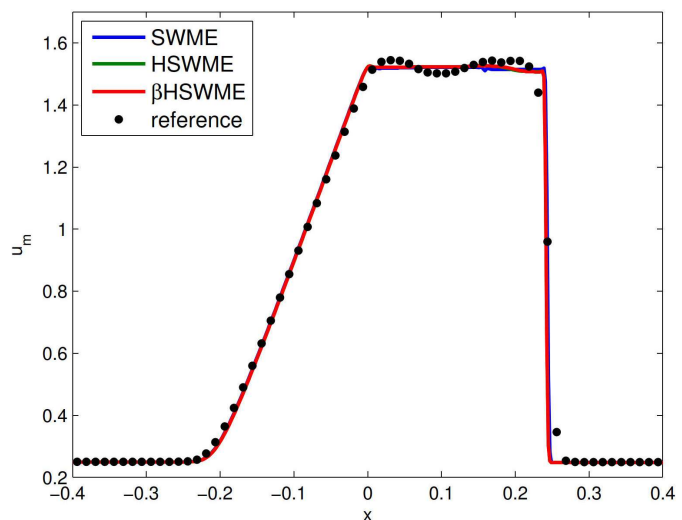

(d) $u_{m}, t=0.1$

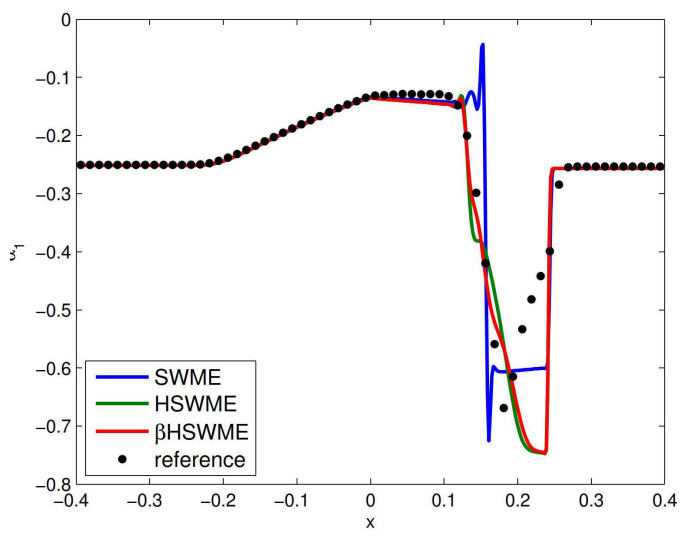

(f) $\alpha_{1}, t=0.1$

Figure 9: Unstable dam break simulation of SWME, HSWME, $\beta$-HSWME for $N=3$ and different times. Reference solution with settings from [27]. 
not completely removed in the new hyperbolic models due to the changed behavior explained in Section 3.3. The $\beta$-HSWME model largely coincides with the HSWME model but indeed leads to an even smoother $\alpha_{1}$ as visible in Fig. 9(f) and again consistent with the interpretation in Section 3.3.

As a summary of the test case we can say that both the HSWME and $\beta$-HSWME model succeed in reducing the oscillations of the SWME model and yield accurate results for this particularly difficult test case.

\subsection{Simulation of a smooth periodic wave}

Next, we focus on a smooth test case simulating water waves moving in a periodic domain, repeating the test setup in [27], given in Table 6 with linear velocity profile.

Table 6: Simulation setup for smooth periodic wave test case.

\begin{tabular}{||c|l||}
\hline friction coefficient & $\lambda=0.1$ \\
\hline slip length & $v=0.1$ \\
\hline temporal domain & $t \in[0,2]$ \\
\hline spatial domain & periodic $x \in[-1,1]$ \\
\hline spatial resolution & $n_{x}=1000$ \\
\hline initial height & $h(x)=1+\exp (3 \cos (\pi(x+0.5))) / \exp (4)$ \\
\hline initial velocity & $u(0, x, \zeta)=0.25 \zeta$ \\
\hline CFL number & $\approx 0.7$ \\
\hline numerical scheme & UPRICE implementation $[21,36]$ \\
\hline
\end{tabular}

After the start the initial bump will lead to two traveling waves, moving left and right, respectively, and then colliding with the respective waves from the other side of the periodic domain. We compare the results at $t_{\text {end }}=2$ when the waves have collided twice. A reference solution using a vertically resolved shallow water model with piecewise constant discretization in $z$-direction is taken from [27].

The results of the smooth test case are shown in Fig. 10 for variables $h$ and $u_{m}$ and all models up to $N=4$ for SWME, HSWME and $\beta$-HSWME. It is evident from this figure that all models seem to converge. Increasing $N$ leads to more accurate simulations and the solution already shows very good agreement with the reference solution, starting from $N=2$ for all models. This shows that the HSWME and the $\beta$-HSWME succeed in the accurate description of this test case, despite their changes and simplifications. Even for larger $N$ the new hyperbolic models do not result in visibly bad accuracy.

Taking a closer look at a direct comparison of the models in Fig. 11, exemplarily for $N=4$, we see no differences in $h$ and $u_{m}$, whereas $\alpha_{1}$ shows a small deviation from the reference solution for HSWME and $\beta$-HSWME (on top of each other). We conclude that the changes in the terms of the HSWME and $\beta$-HSWME models do not influence the 


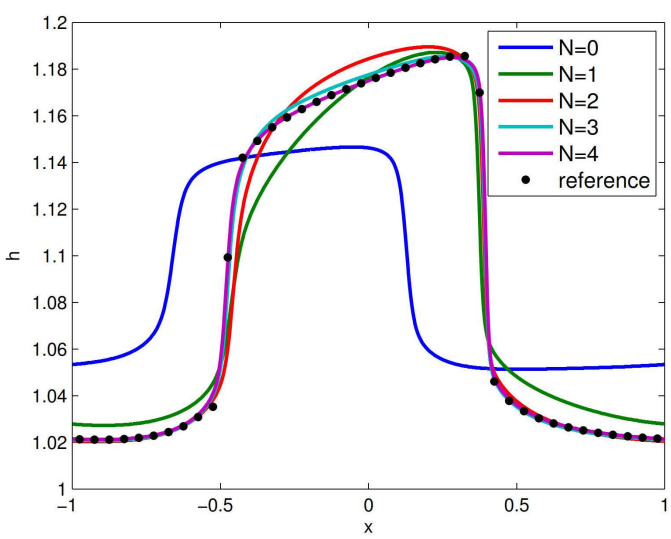

(a) SWME, $h$

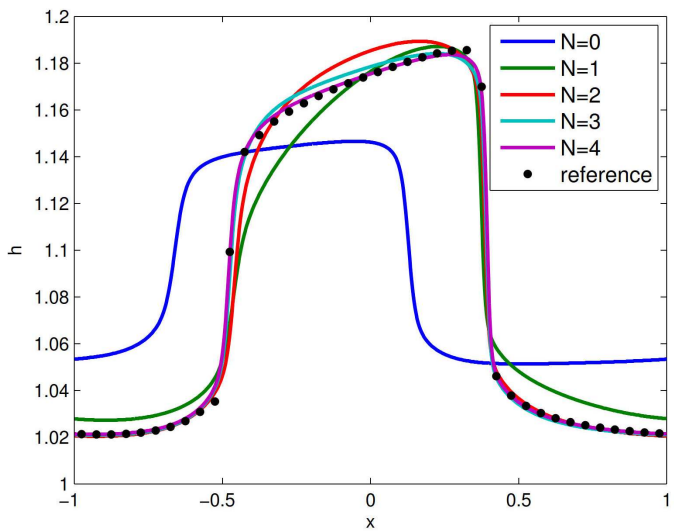

(c) HSWME, $h$

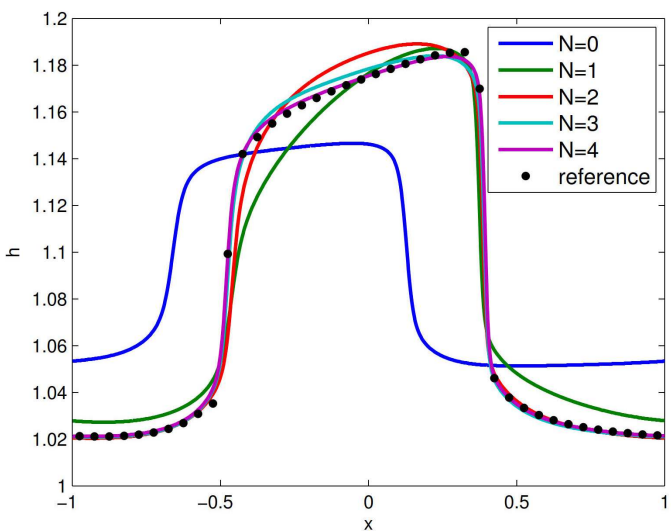

(e) $\beta$-HSWME, $h$

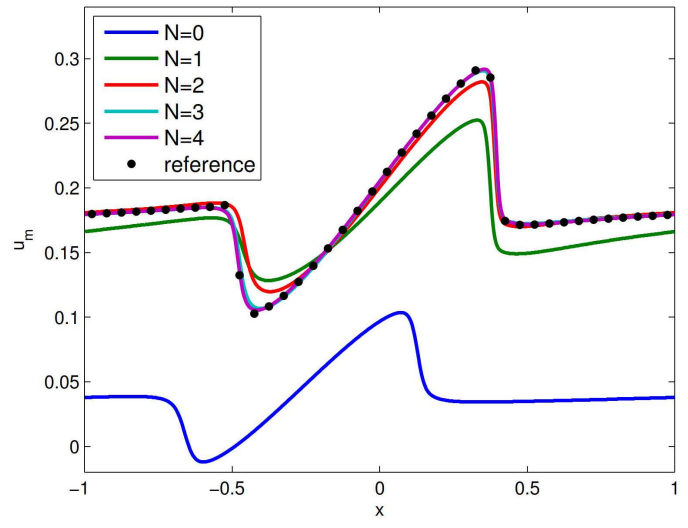

(b) SWME, $u_{m}$

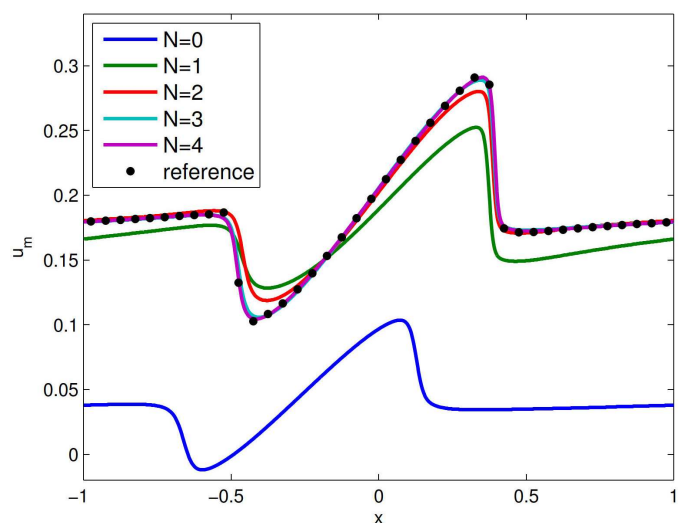

(d) HSWME, $u_{m}$

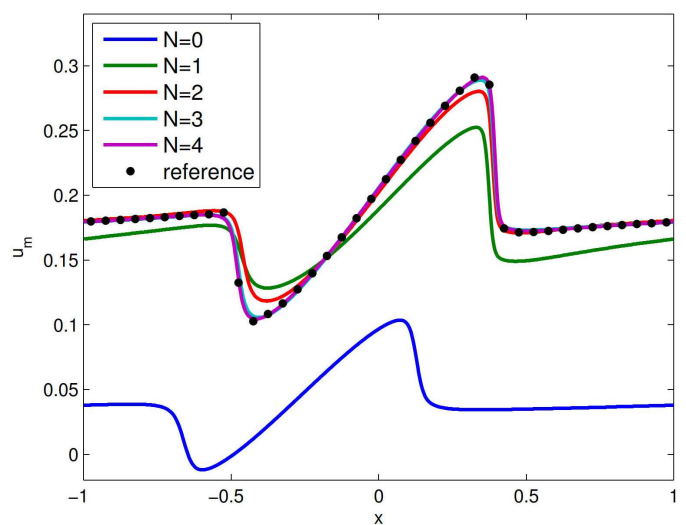

(f) $\beta$-HSWME, $u_{m}$

Figure 10: Smooth test case from [27] for SWME, HSWME and $\beta$-HSWME for varying $N$ and $t=2$. 


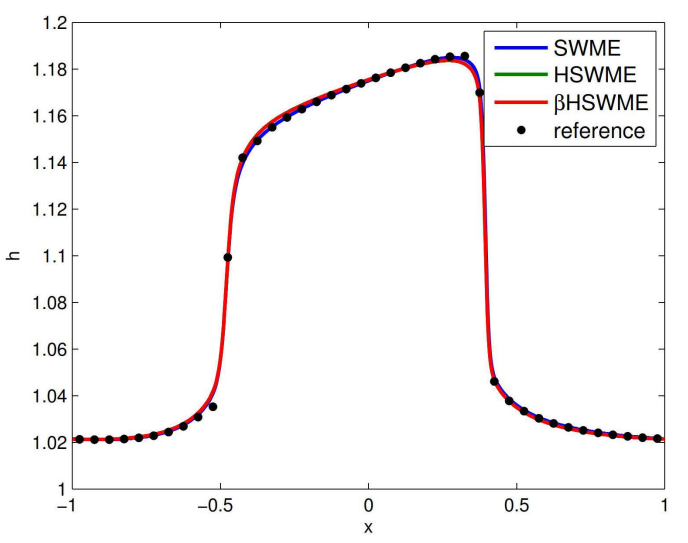

(a) $h$

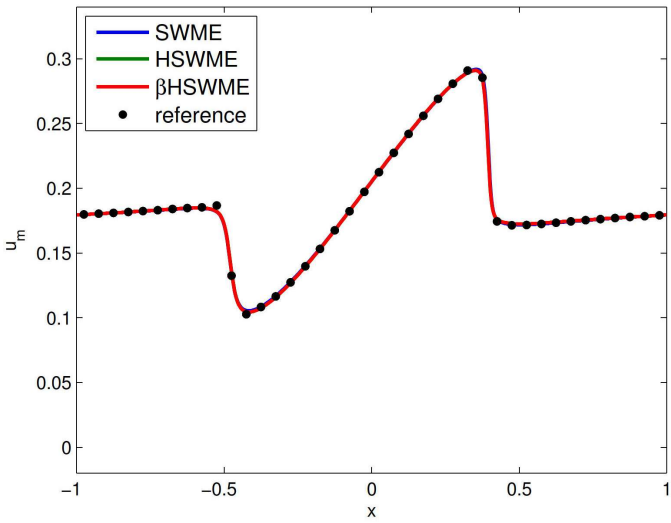

(b) $u$

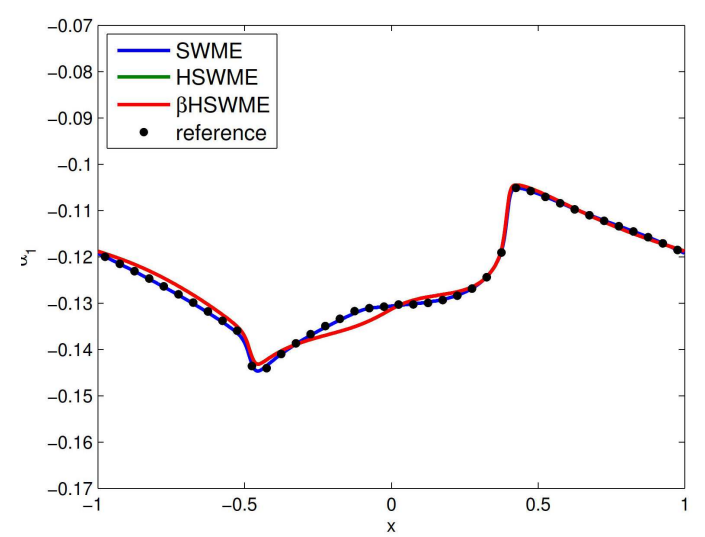

(c) $\alpha_{1}$

Figure 11: Smooth test case from [27] for SWME, HSWME and $\beta$-HSWME, $N=4$ and $t=2$.

quality of the solution for this test case. The hyperbolic models successfully approximate the macroscopic variables $h$ and $u_{m}$ with no differences.

In Fig. 12, we display the convergence of the relative error in water height and velocity with increasing $N$. We can clearly see the error reduction with respect to increasing $N$ throughout all models. We note that for $N=0,1$ all models are equal and thus result in the same error, as depicted. For $N \geq 3$, the SWME model results in a slightly smaller error. However, the hyperbolic models also yield reduced errors. It is expected that the linearization for the HSWME model leads to some small additional model errors that can especially be seen for larger $N$, where the SWME model benefits from its full nonlinearity depending on more variables. However, the global hyperbolicity of HSWME and $\beta$ HSWME might counteract this effect, especially for $N=2$, where the SWME in fact yields a slightly larger error. The overall error is below $1 \%$ for the higher order models and thus very small, especially in comparison to the standard shallow water model $N=0$ that 


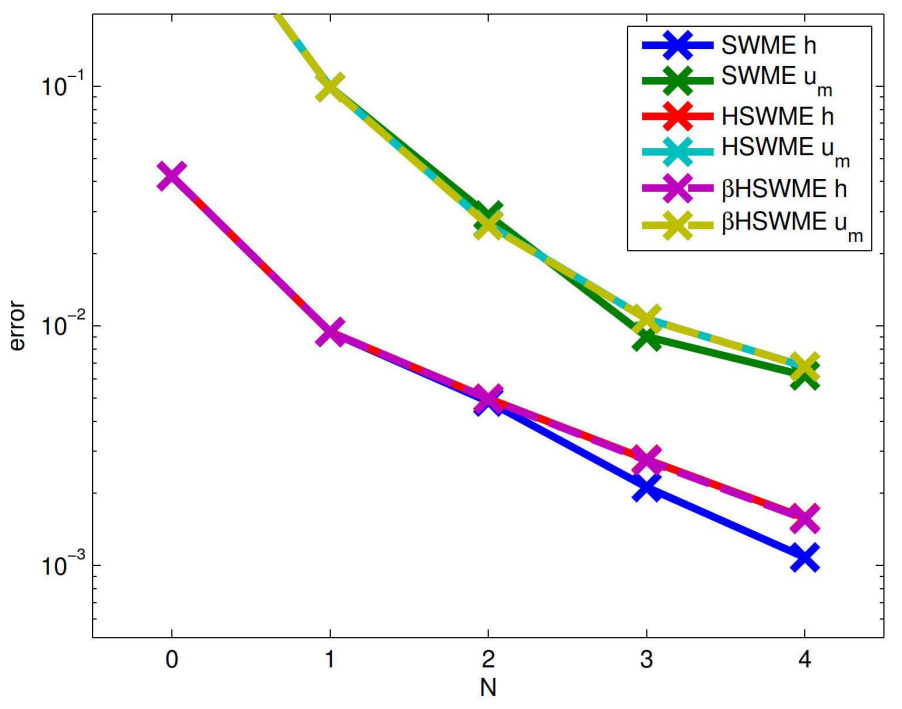

Figure 12: Error convergence of smooth test case from [27] for SWME, HSWME and $\beta$-HSWME.

leads to errors in the order of $10 \%$ for the height $h$ and the mean velocity $u_{m}$, respectively.

As a summary of this smooth test case we can say that both the HSWME and $\beta$ HSWME model converge to the same solution as the original SWME model and especially the water height and mean velocity show no visual difference. We remark that despite the changes in the equations, the model error is almost the same for all models. This means that the moment model is in general not sensitive to changes of the equations and the moment approach thus seems a good approach for modeling shallow water flows.

\subsection{Simulation of dam break problem}

Since the new HSWME and $\beta$-HSWME showed good behavior for a periodic test case and smooth initial conditions, we want to investigate if the models also converge for discontinuous initial conditions, which typically pose more difficulties, especially for non-conservative systems. We show results for a dam break problem, where an initial discontinuity is propagated through the domain.

The test setup in Table 7 is similar to the case with demonstrated instabilities, but we choose a stable test case to allow for fair comparison with the SWME model.

Fig. 13 shows the results of the dam break problem for $h, u_{m}$ and $\alpha_{1}$, all models and order up to $N=5$. In the left column, we see convergence of the SWME model, whereas the right column shows the HSWME results. The $\beta$-HSWME largely coincide with the HSWME model and are thus not shown in this figure.

As before both the SWME and HSWME models show good numerical results compared to the reference solution of a vertically resolved system, computed with the software from [27]. The accuracy is improved by increasing the number of moments, starting 


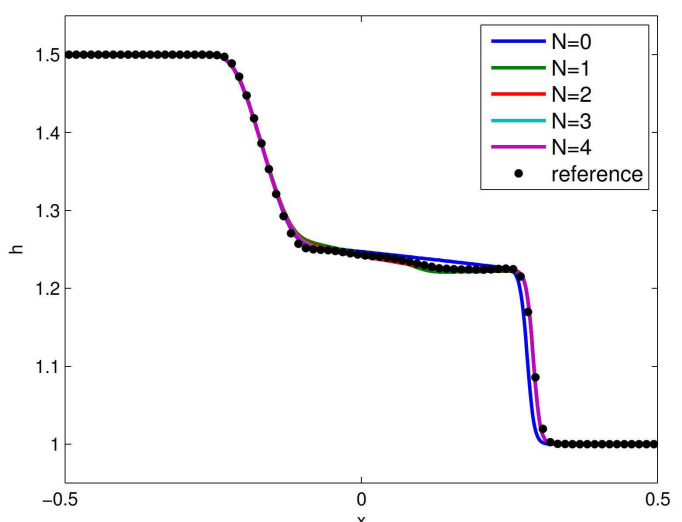

(a) SWME, $h$

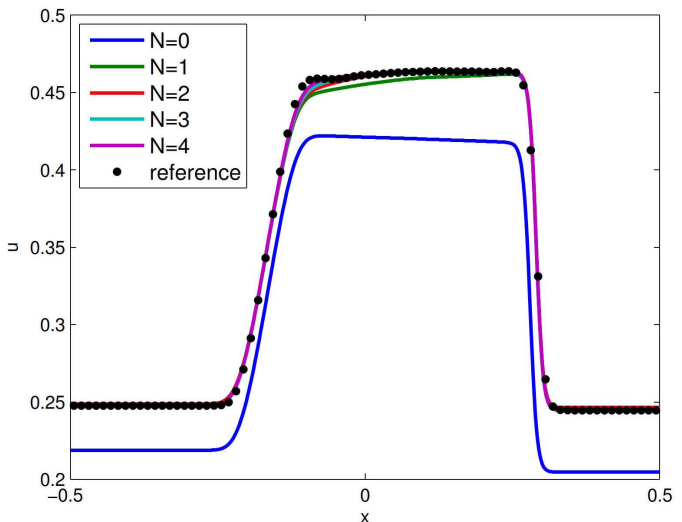

(c) SWME, $h$

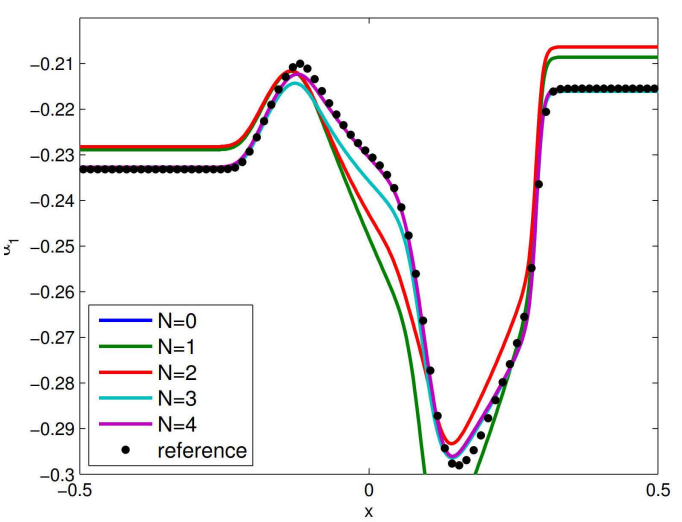

(e) SWME, $\alpha_{1}$

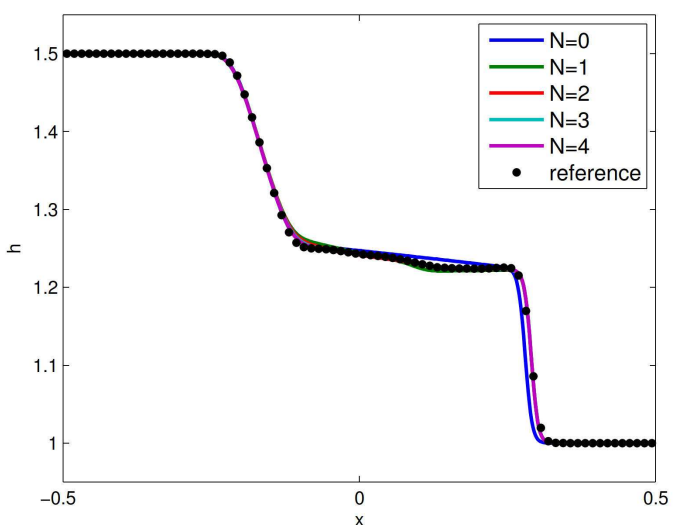

(b) HSWME, $u_{m}$

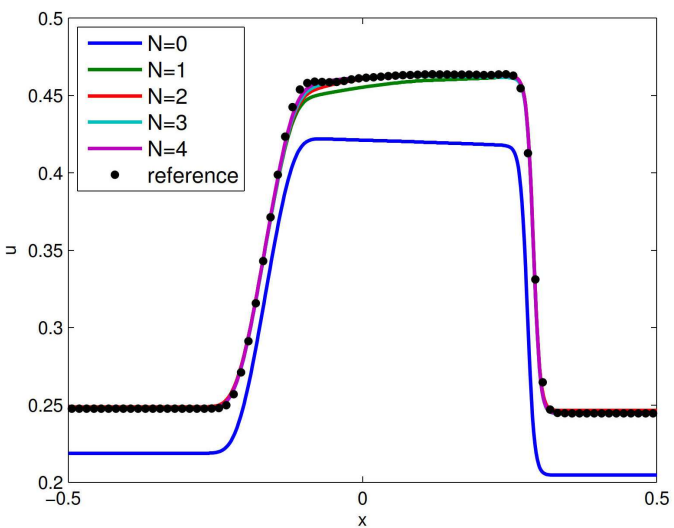

(d) HSWME, $u_{m}$

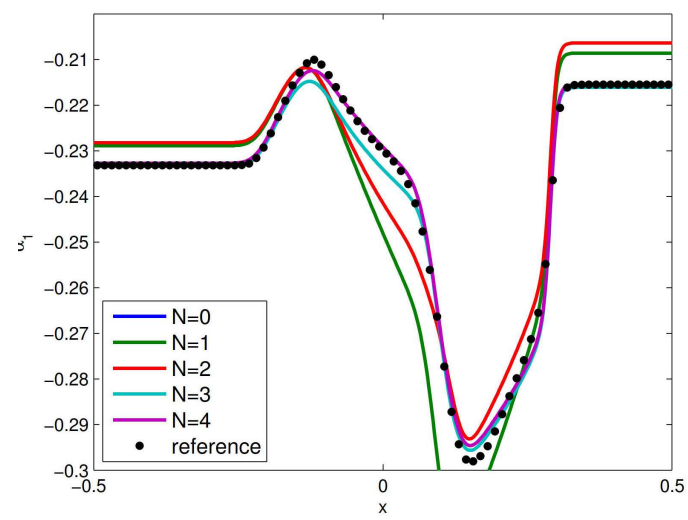

(f) HSWME, $\alpha_{1}$

Figure 13: Small dam break test case for SWME and HSWME ( $\beta$-HSWME practically identical), varying $N$ and $t=0.2$. 
Table 7: Simulation setup for dam break test case.

\begin{tabular}{||c|l||}
\hline friction coefficient & $\lambda=0.1$ \\
\hline slip length & $v=0.1$ \\
\hline spatial domain & $x \in[-1,1]$ \\
\hline spatial resolution & $n_{x}=1000$ \\
\hline initial height & $h(x)= \begin{cases}1.5 & \text { if } x \leq 0, \\
1 & \text { if } x>0\end{cases}$ \\
\hline initial velocity & $u(0, x, \zeta)=0.25 \zeta$ \\
\hline CFL number & $\approx 0.7$ \\
\hline numerical scheme & UPRICE implementation [21,36] \\
\hline
\end{tabular}

with $N=1$. The fact that we observe no visual difference between the three models again indicates that the changes introduced by the HSWME and the $\beta$-HSWME do not have a large influence on the accuracy of the model while being sufficient to guarantee hyperbolicity.

Fig. 14 shows the relative error in water height and velocity for this test case. We can clearly see a fast error reduction with increasing order $N$ for all involved models. Again we note that all models are equivalent for $N=0,1$. For a larger number of moments the accuracy of the new hyperbolic models is the same as for the SWME, while still reducing the error below $1 \%$ in contrast to the SWE model $N=0$, which shows a

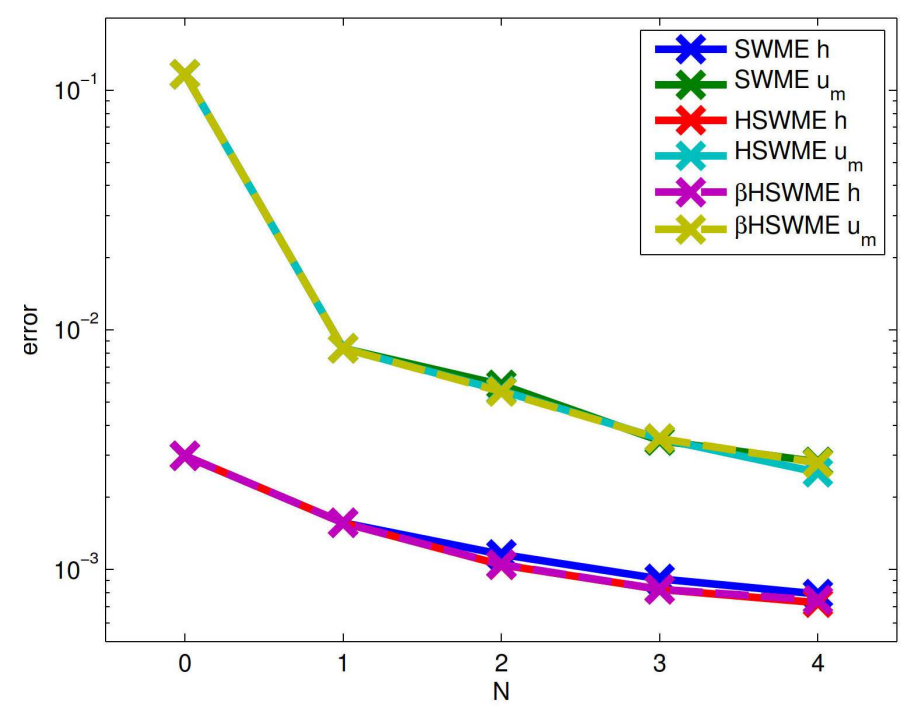

Figure 14: Error convergence of dam break test case for SWME, HSWME and $\beta$-HSWME. 
substantially larger error. We thus see that the new hyperbolic models succeed in yielding the desired accuracy while preserving the hyperbolicity of the model. However, the differences in the higher order results are smaller than in the smooth test case from the previous section. This is despite the discontinuity in the initial data. Again it seems that the moment method in general seems to be quite robust when it comes to slight changes of the equations, which increases usability and credibility of the approach.

As a summary of this test case we can say that also for discontinuous data the models seem to converge to the reference solution. All models yield relatively similar results in this case which hints at the fact that the moment method itself seems to be very robust in this application.

\section{Conclusion}

In this paper we successfully derived hyperbolic moment approximations of shallow water flows for higher orders that yield good accuracy compared to existing models and reference solutions as demonstrated by numerical simulations.

After a brief introduction the derivation of the SWME was reviewed and we showed the lack of hyperbolicity, a breakdown of hyperbolicity for the SWME in simulations using an explicit example as initial state, and pointed out arising instabilities in a numerical solution.

Using this motivation we introduced the hyperbolic shallow water moment equations HSWME, based on a linearization of the system matrix around a linear deviation from equilibrium. After a detailed analysis, we could prove global hyperbolicity even for larger number of equations and give the analytical form of the equations including formulas for the eigenvalues.

Based on the HSWME, we allowed more flexibility by deriving a second hyperbolic model called $\beta$-HSWME as a generalization of the HSWME. We gave an algorithm, so that for a given set of real and distinct eigenvalues there exists a unique solution for the parameters $\beta_{i}$ so that the system matrix adopts these eigenvalues and hence becomes hyperbolic for all orders $N \in \mathbb{N}$. We provide a physical interpretation of the new HSWME and $\beta$-HSWME in comparison to the SWME model and linear stability of all moment models was shown in a domain around equilibrium.

We could show that the new hyperbolic models successfully remove the oscillations observed in the numerical solutions before. In two additional simulations of a smooth periodic test case and an initially discontinuous dam break test case, we could show the good agreement of the solution of our new hyperbolic models HSWME and $\beta$-HSWME in comparison with the existing SWME and a reference solution. With increasing the number of moments we saw an error reduction and even smaller errors than for the SWME for some cases.

We conclude that the new HSWME and $\beta$-HSWME are two successful new models for the simulation of complex shallow flows that allow for variations of the vertical velocity 
while providing necessary global hyperbolicity and still resulting in good numerical accuracy together with significant speedup with respect to the reference model.

Despite the changed equations, all models yield similar results which means that the moment method in general seems to be quite robust, which increases its usability and credibility.

Further work should focus on extension of the hyperbolic shallow water moment models to other applications, e.g. granular flows or multi-dimensional problems. Another possibility could be the investigation of the shallow water moment equations for strongly enforced boundary conditions. Due to the general form of the $\beta$-HSWME it could be beneficial to explore different choices of propagation speeds to obtain suitable models.

\section{Acknowledgments}

The authors thank the referees for their constructive and helpful comments.

The first author was funded by a joint postdoctoral scholarship from Freie Universität Berlin and Peking University.

\section{Appendices}

\section{A Proof of explicit HSWME system matrix $A_{H}$}

Theorem A.1. The HSWME system matrix $A_{H}$ is given by

$$
A_{H}=\left(\begin{array}{cccccc}
g h-u_{m}^{2}-\frac{1}{3} \alpha_{1}^{2} & 2 u_{m} & \frac{2}{3} \alpha_{1} & & & \\
-2 u_{m} \alpha_{1} & 2 \alpha_{1} & u_{m} & \frac{3}{5} \alpha_{1} & & \\
-\frac{2}{3} \alpha_{1}^{2} & & \frac{1}{3} \alpha_{1} & u_{m} & \ddots & \\
& & & \ddots & \ddots & \frac{N+1}{2 N+1} \alpha_{1}
\end{array}\right),
$$

where all other entries are zero.

Proof. The Jacobian matrix $A_{H}$ is computed by taking the derivative of the flux function, subtracting the left-hand side non-conservative terms, and then setting the higher order moments to zero. The proof will consider the mass and momentum equation and the equations for $\alpha_{i}$ separately. The latter are further divided into the non-conservative and conservative parts. 


\section{The mass and momentum equations}

The first two rows of the coefficient matrix corresponding to mass and momentum balance equations can be easily determined as

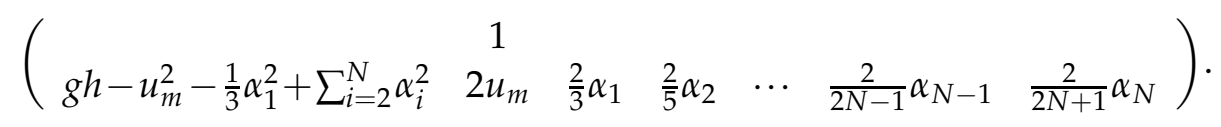

When setting all the higher moments to zero, the remaining part is given by

$$
\left(\begin{array}{ccc}
g h-u_{m}^{2}-\frac{1}{3} \alpha_{1}^{2} & 2 u_{m} & \frac{2}{3} \alpha_{1}
\end{array}\right)
$$

\section{The equations for $\alpha_{i}$}

We first show the general structure of this part of the matrix.

Recall that the $(i+2)^{t h}$ equation of the system with $i \geq 1$ is given by (2.6)

$$
\frac{\partial\left(h \alpha_{i}\right)}{\partial t}+\frac{\partial \tilde{F}_{i}}{\partial x}=\tilde{Q}_{i}+P_{i}
$$

The system matrix $A_{H}$ is thus composed of terms from $\frac{\partial \tilde{F}_{i}}{\partial x}$ and $\tilde{Q}_{i}$. We will consider the two terms separately.

\subsection{The conservative part $\frac{\partial \tilde{F}_{i}}{\partial x}$}

Recall from (2.7) that

$$
\tilde{F}_{i}=h 2 u_{m} \alpha_{i}+h \sum_{j, k=1}^{N} \underbrace{(2 i+1) \int_{0}^{1} \phi_{i} \phi_{j} \phi_{k} \mathrm{~d} \zeta}_{B_{i j k}} \alpha_{j} \alpha_{k} .
$$

We further consider the first term $h 2 u_{m} \alpha_{i}$ and the second term including $B_{i j k}$ separately.

Using all $\alpha=\left\{\alpha_{1}, \cdots \alpha_{N}\right\}$ and setting all higher variables $\alpha_{i}=0$, for $i \geq 2$ in the coefficient matrix of the first term leads to

$$
\frac{\partial\left(2 h u_{m} \boldsymbol{\alpha}\right)}{\partial V}=\left(\begin{array}{ccccc}
-2 u_{m} \alpha_{1} & 2 \alpha_{1} & 2 u_{m} & & \\
& & & \ddots & \\
& & & & 2 u_{m}
\end{array}\right) \text {. }
$$

Now we will consider the second term

$$
h \sum_{j, k=1}^{N} \underbrace{(2 i+1) \int_{0}^{1} \phi_{i} \phi_{j} \phi_{k} \mathrm{~d} \zeta}_{B i j k} \alpha_{j} \alpha_{k} .
$$


We will first simplify this the integral over the Legendre polynomials and than again take the derivative with respect to the conservative variables $V$.

Therefore, assume $j=1$. For the triple Legendre integral, we use that if $i+j<k$ or $i+k<j$ or $j+k<i$, then

$$
\int_{0}^{1} \phi_{i} \phi_{j} \phi_{k} \mathrm{~d} \zeta=0
$$

For the proof see e.g. [17]. Let $i$ be fixed, then from $i+1<k$ and $i+k<1$ and $1+k<i$ it follows that only for $i-1 \leq k \leq i+1$, the integral can become non-zero. Therefore, we take a closer look at the three cases, $k=i, k+1=i$ and $k-1=i$.

For the first case $k=i$ the integral is zero. This is because in [17] it is shown that the integral vanishes if $(j+i+k) \bmod 2 \neq 0$. For $j=1$ that precisely happens for the first case of $k=i$ since then $(j+i+k) \bmod 2=(1+2 k) \bmod 2=1$. Therefore, if $j=1$, only case 2 (i.e $k+1=i)$ and case 3 (i.e. $k-1=i$ ) need to be considered.

The same can be shown for $k=1$ and an arbitrary $j$ analogously due to commutativity of the sum and the integral.

Using this, we can pull out the second and third case from the sum. This results in

$$
\begin{aligned}
h \sum_{j, k=1}^{N}(2 i+1) \int_{0}^{1} \phi_{i} \phi_{j} \phi_{k} \mathrm{~d} \zeta \alpha_{j} \alpha_{k}= & h \underbrace{\sum_{j, k=2}^{N} B_{i j k} \alpha_{j} \alpha_{k}}_{\text {first term }} \\
& +\underbrace{2 B_{i, 1, i-1} h \alpha_{1} \alpha_{i+1}}_{\text {second term }}+\underbrace{2 B_{i, 1, i+1} h \alpha_{1} \alpha_{i-1}}_{\text {third term }} .
\end{aligned}
$$

Notice that due to the commutativity of the integral and multiplication of the $\alpha_{i}$ for the second term $B_{i, 1, i+1} h \alpha_{1} \alpha_{i-1}=B_{i, i+1,1} h \alpha_{i-1} \alpha_{1}$ and analogously for the third term. Therefore, the factor 2 appears.

To obtain the entries of our system matrix, we take the derivative of all three terms of the right hand side with respect to $h, h u_{m}$, and $\left(h \alpha_{l}\right)$ for $l \geq 1$ :

1. Consider the first term of (A.3):

$$
\frac{\partial\left(h \sum_{j, k=2}^{N} B_{i j k} \alpha_{j} \alpha_{k}\right)}{\partial\left(h \alpha_{l}\right)}=\sum_{j, k=2}^{N} B_{i j k} \frac{\partial\left(h \alpha_{j} \alpha_{k}\right)}{\partial\left(h \alpha_{l}\right)}=\sum_{j, k=2}^{N} B_{i j k}\left(\alpha_{j} \delta_{k, l}+\alpha_{k} \delta_{j, l}\right),
$$

with $\delta_{i, j}$ being the Kronecker delta. One can see that for every $i, j$ and $k$, these terms always depend on $\alpha_{m}$ with $m \geq 2$ or are equal to zero. The same can be observed when taking the derivative with respect to $h$ and to $h u_{m}$. Therefore all terms reduce to 0 when setting all $\alpha_{m}=0$. Similarly, all terms reduce to zero when taking the derivative with respect to $h$ and $h u_{m}$ and setting $\alpha_{m}=0$.

2. Consider the second term of (A.3):

$$
\frac{\partial\left(2 B_{i, l, i-1} h \alpha_{1} \alpha_{i-1}\right)}{\partial\left(h \alpha_{l}\right)}=2 B_{i, l, i-1} \alpha_{i-1} \delta_{1, l}+2 B_{i, l, i-1} \alpha_{1} \delta_{i-1, l}
$$


Notice that $B_{i, l, i-1} \alpha_{i-1} \delta_{1, l}$ vanishes for $i>2$ when setting $\alpha_{i}=0$ for $i \geq 2$. Furthermore, when taking the derivative with respect to $h$ and $h u_{m}$ from $B_{i, l, i-1} \alpha_{i-1} \delta_{1, l}$ only one additional non-zero term appears in the first column of the system matrix. This non-zero term evaluates to $-\frac{2}{3} \alpha_{1}^{2}$. All other terms depend on $\alpha_{i}$ with $i \geq 2$ and are therefore set to zero.

The term $B_{i, l, i-1} \alpha_{1} \delta_{i-1, l}$ will only be non-zero for $i-1=l$. Leading to a non-zero entry on the first lower diagonal, which we call $a_{i}^{F}$.

The entry $a_{i}^{F}$ can be computed for $i=2, \cdots, N$ using orthogonality and recursion formulas of the Legendre polynomials by

$$
\begin{aligned}
a_{i}^{F} & =2(2 i+1) \int_{0}^{1} \phi_{i} \phi_{1} \phi_{i-1} \mathrm{~d} \zeta \alpha_{1} \\
& =2(2 i+1) \int_{0}^{1} \phi_{i}(1-2 \zeta) \phi_{i-1} \mathrm{~d} \zeta \alpha_{1} \\
& =\frac{2 i}{2 i-1} \alpha_{1} .
\end{aligned}
$$

3. The third term of (A.3) leads to a corresponding entry on the first upper diagonal, which we call $c_{i+1}^{F}$. Analogously, this entry $c_{i+1}^{F}$ can be computed for $i=1, \cdots, N-1$ using orthogonality and recursion formulas of the Legendre polynomials by

$$
\begin{aligned}
c_{i+1}^{F} & =2(2 i+1) \int_{0}^{1} \phi_{i} \phi_{1} \phi_{i+1} \mathrm{~d} \zeta \alpha_{1} \\
& =2(2 i+1) \int_{0}^{1} \phi_{i}(1-2 \zeta) \phi_{i+1} \mathrm{~d} \zeta \alpha_{1} \\
& =\frac{2 i+2}{2 i+3} \alpha_{1} .
\end{aligned}
$$

Together with the mass and momentum equation of (A.1) and the results from the first term (A.2) we get for the coefficient matrix $\frac{\partial F}{\partial V}{ }_{\text {mod }}$ :

$$
\frac{\partial F}{\partial V}_{\text {mod }}=\left(\begin{array}{cccccc}
g h-u_{m}^{2}-\frac{1}{3} \alpha_{1}^{2} & 2 u_{m} & \frac{2}{3} \alpha_{1} & & & \\
-2 u_{m} \alpha_{1} & 2 \alpha_{1} & 2 u_{m} & \frac{4}{5} \alpha_{1} & & \\
-\frac{2}{3} \alpha_{1}^{2} & & \frac{4}{3} \alpha_{1} & 2 u_{m} & \ddots & \\
& & & \ddots & \ddots & \frac{2 N}{2 N+1} \alpha_{1}
\end{array}\right) .
$$

\subsection{The non-conservative part $\tilde{Q}_{i}$}

Recalling (2.8), the $(i+2)^{\text {th }}$ row uses 


$$
\tilde{Q}_{i}=u_{m} \frac{\partial\left(h \alpha_{i}\right)}{\partial x}-\sum_{j, k=1}^{N} \underbrace{(2 i+1) \int_{0}^{1} \phi_{i}^{\prime}\left(\int_{0}^{\zeta} \phi_{j} \mathrm{~d} \hat{\zeta}\right) \phi_{k} \mathrm{~d} \zeta}_{C_{i j k}} \cdot \alpha_{k} \frac{\partial\left(h \alpha_{j}\right)}{\partial x},
$$

and we investigate the first and the second term separately. The first term obviously leads to the term $u_{m}$ on the diagonal of $Q$.

We can simplify the second term of $\tilde{Q}_{i}$ for the case $\alpha_{i}=0$, for $i \geq 2$, since it is multiplied with $\frac{\left(h \alpha_{j}\right)}{\partial x} \alpha_{k}$. Independently of $j$, each term will become zero for $k \neq 1$, since all $\alpha_{k}=0$ for $2 \leq k \leq N$. Therefore, only the term

$$
\sum_{j}^{N} \underbrace{(2 i+1) \int_{0}^{1} \phi_{i}^{\prime}\left(\int_{0}^{\zeta} \phi_{j} \mathrm{~d} \hat{\zeta}\right) \phi_{1} \mathrm{~d} \zeta}_{C_{i j 1}} \frac{\partial\left(h \alpha_{j}\right)}{\partial x} \alpha_{1}
$$

needs to be considered.

Analogously to the previous conservative part, it turns out that $C_{i j 1}=0$ for $|i-j| \neq 1$ and $B_{i j 1} \neq 0$ for $|i-j|=1$ except for the first two rows.

Since $\tilde{Q}_{i}$ does not depend on any derivative with respect to $h$ and $u_{m}$, the first two columns of $Q$ are always zero. The first two rows of $Q$ will also be zero, which consistently means that the mass and momentum equations do not contain non-conservative terms.

We thus get one non-zero term $c_{i+1}^{Q}$ on the first upper diagonal and one term $a_{i}^{Q}$ on the first lower diagonal, as follows:

$$
\begin{aligned}
a_{i}^{Q} & =(2 i+1) \int_{0}^{1} \phi_{i}^{\prime}\left(\int_{0}^{\zeta} \phi_{i-1} \mathrm{~d} \hat{\zeta}\right) \phi_{1} \mathrm{~d} \zeta \alpha_{1} \\
& =\frac{i+1}{2 i-1} \alpha_{1}
\end{aligned}
$$

and

$$
\begin{aligned}
c_{i+1}^{Q} & =(2 i+1) \int_{0}^{1} \phi_{i}^{\prime}\left(\int_{0}^{\zeta} \phi_{i+1} \mathrm{~d} \hat{\zeta}\right) \phi_{1} \mathrm{~d} \zeta \alpha_{1} \\
& =\frac{i}{2 i+3} \alpha_{1} .
\end{aligned}
$$

This results in the following $Q_{\text {mod }}$ :

$$
Q_{\text {mod }}=\left(\begin{array}{cccc} 
& & & \\
u_{m} & \frac{1}{5} \alpha_{1} & & \\
\frac{3}{3} \alpha_{1} & u_{m} & \ddots & \\
& \ddots & \ddots & \frac{N-1}{2 N+1} \alpha_{1}
\end{array}\right) .
$$


The system matrix is then given by

$$
A_{H}={\frac{\partial F}{\partial V_{m o d}}}_{-Q_{\text {mod }}}
$$

which completes the proof.

\section{B Proof of characteristic polynomial of HSWME matrix $A_{H}$}

Theorem B.1. The HSWME system matrix $A_{H} \in \mathbb{R}^{(N+2) \times(N+2)}$ (3.1) has the following characteristic polynomial

$$
\chi_{A}(\lambda)=\left(\left(\lambda-u_{m}\right)^{2}-g h-\alpha_{1}^{2}\right) \cdot \chi_{A_{2}}\left(\lambda-u_{m}\right),
$$

where the matrix $A_{2} \in \mathbb{R}^{N \times N}$ is defined as follows

$$
A_{2}=\left(\begin{array}{cccc} 
& c_{2} & & \\
a_{2} & & \ddots & \\
& \ddots & & c_{N} \\
& & a_{N} &
\end{array}\right),
$$

with values $c_{i}=\frac{i+1}{2 i+1} \alpha_{1}$ and $a_{i}=\frac{i-1}{2 i-1} \alpha_{1}$ the values above and below the diagonal, respectively, from (3.1).

Proof. We write $A_{H}=\widetilde{A}_{H}+u_{m} I$ and $\widetilde{\lambda}=\lambda-u_{m}$, so that we can compute the characteristic polynomial using

$$
\begin{aligned}
\chi_{A}(\lambda) & =\operatorname{det}\left(A_{H}-\lambda I\right) \\
& =\operatorname{det}\left(\widetilde{A}_{H}-\left(\lambda-u_{m}\right) I\right) \\
& =\operatorname{det}\left(\widetilde{A}_{H}-\widetilde{\lambda} I\right) \\
& =:\left|\widetilde{A}_{H}-\widetilde{\lambda} I\right| .
\end{aligned}
$$

We use the following notation for conciseness:

$$
\begin{aligned}
& d_{1}=g h-u_{m}^{2}-\frac{1}{3} \alpha_{1}^{2}, \quad d_{2}=\frac{2}{3} \alpha_{1} \\
& d_{3}=-2 u_{m} \alpha_{1}, \quad d_{4}=2 \alpha_{1} \\
& d_{5}=-\frac{2}{3} \alpha_{1}^{2}, \quad a_{2}=\frac{1}{3} \alpha_{1}
\end{aligned}
$$


and compute $\left|\widetilde{A}_{H}-\widetilde{\lambda} I\right|$ by always developing with respect to the first row

$$
\begin{aligned}
& \left|\widetilde{A}_{H}-\widetilde{\lambda} I\right|=\left|\begin{array}{cccccc}
-\widetilde{\lambda}-u_{m} & 1 & & & & \\
d_{1} & -\widetilde{\lambda}+u_{m} & d_{2} & & & \\
d_{3} & d_{4} & -\widetilde{\lambda} & c_{2} & & \\
d_{5} & & a_{2} & -\widetilde{\lambda} & \ddots & \\
& & & \ddots & \ddots & c_{N} \\
& & & & a_{N} & -\widetilde{\lambda}
\end{array}\right| \\
& =\left(-\widetilde{\lambda}-u_{m}\right)\left|\begin{array}{ccccc}
-\widetilde{\lambda}+u_{m} & d_{2} & & & \\
d_{4} & -\widetilde{\lambda} & c_{2} & & \\
& a_{2} & -\widetilde{\lambda} & \ddots & \\
& & \ddots & \ddots & c_{N} \\
& & & a_{N} & -\widetilde{\lambda}
\end{array}\right|-1\left|\begin{array}{ccccc}
d_{1} & d_{2} & & & \\
d_{3} & -\widetilde{\lambda} & c_{2} & & \\
d_{5} & a_{2} & -\widetilde{\lambda} & \ddots & \\
& & \ddots & \ddots & c_{N} \\
& & & a_{N} & -\widetilde{\lambda}
\end{array}\right| \\
& =\left(-\tilde{\lambda}-u_{m}\right)\left(\left(-\widetilde{\lambda}+u_{m}\right)\left|\begin{array}{cccc}
-\widetilde{\lambda} & c_{2} & & \\
a_{2} & -\widetilde{\lambda} & \ddots & \\
& \ddots & \ddots & c_{N} \\
& & a_{N} & -\widetilde{\lambda}
\end{array}\right|-d_{2}\left|\begin{array}{cccc}
d_{4} & c_{2} & & \\
& -\widetilde{\lambda} & \ddots & \\
& \ddots & \ddots & c_{N} \\
& & a_{N} & -\widetilde{\lambda}
\end{array}\right|\right) \\
& -d_{1}\left|\begin{array}{cccc}
-\widetilde{\lambda} & c_{2} & & \\
a_{2} & -\widetilde{\lambda} & \ddots & \\
& \ddots & \ddots & c_{N} \\
& & a_{N} & -\widetilde{\lambda}
\end{array}\right|+d_{2}\left|\begin{array}{cccc}
d_{3} & c_{2} & & \\
d_{5} & -\widetilde{\lambda} & \ddots & \\
& \ddots & \ddots & c_{N} \\
& & a_{N} & -\widetilde{\lambda}
\end{array}\right| \\
& =\left(-\widetilde{\lambda}-u_{m}\right)\left(\left(-\widetilde{\lambda}+u_{m}\right)\left|A_{2}\right|-d_{2} d_{4}\left|A_{3}\right|\right)-d_{1}\left|A_{2}\right|+d_{2}\left(d_{3}\left|A_{3}\right|-c_{2} d_{5}\left|A_{4}\right|\right) \text {, }
\end{aligned}
$$

where $A_{i}$ are the following submatrices

$$
A_{i}=\left|\begin{array}{cccc}
-\widetilde{\lambda} & c_{i} & & \\
a_{i} & -\widetilde{\lambda} & \ddots & \\
& \ddots & \ddots & c_{N} \\
& & a_{N} & -\widetilde{\lambda}
\end{array}\right| .
$$

From (B.2), we directly obtain the recursion formula

$$
\left|A_{4}\right|=\frac{1}{a_{2} c_{2}}\left(\left|A_{2}\right|+\widetilde{\lambda}\left|A_{3}\right|\right),
$$

so that we can eliminate $\left|A_{4}\right|$ from the expression and factorize the remaining terms with 
respect to $\left|A_{2}\right|$ and $\left|A_{3}\right|$ as follows

$$
\begin{aligned}
\chi_{A}(\lambda) & =\left(-\widetilde{\lambda}-u_{m}\right)\left(\left(-\widetilde{\lambda}+u_{m}\right) A_{2}-d_{2} d_{4} A_{3}\right)-d_{1} A_{2}+d_{2}\left(d_{3} A_{3}-c_{2} d_{5} A_{4}\right) \\
& =A_{2}\left(\widetilde{\lambda}^{2}-u_{m}^{2}-d_{1}+\frac{d_{5} d_{2}}{a_{2}}\right)+A_{3}\left(\left(\widetilde{\lambda}+u_{m}\right) d_{2} d_{4}+d_{2} d_{3}+\frac{d_{5} d_{2}}{a_{2}}\right) \\
& =\left|A_{2}\right|\left(\widetilde{\lambda}^{2}-g h-\alpha_{1}^{2}\right)+\left|A_{3}\right|\left(\left(\widetilde{\lambda}+u_{m}\right) 2 \alpha_{1}-u_{m} 2 \alpha_{1}-\widetilde{\lambda} 2 \alpha_{1}\right) \\
& =\left|A_{2}\right|\left(\widetilde{\lambda}^{2}-g h-\alpha_{1}^{2}\right)+\left|A_{3}\right| \cdot 0
\end{aligned}
$$

where we inserted the specific terms for the variables $d_{i}$ and $a_{2}$. Going back to the standard notation $\chi_{A_{2}}=\left|A_{2}\right|$, we finally have

$$
\chi_{A}(\lambda)=\left((\lambda-u)^{2}-g h-\alpha_{1}^{2}\right) \cdot \chi_{A_{2}}(\lambda-u)
$$

which completes the proof.

\section{References}

[1] Remi Abgrall and Smadar Karni. A comment on the computation of non-conservative products. Journal of Computational Physics, 229(8):2759-2763, 2010.

[2] Zhenning Cai, Yuwei Fan, and Ruo Li. Globally hyperbolic regularization of Grad's moment system in one dimensional space. Communications in Mathematical Sciences, 11(2):547-571, 2013.

[3] Zhenning Cai, Yuwei Fan, and Ruo Li. On hyperbolicity of 13-moment system. Kinetic and Related Models, 7(3):415-432, 2014.

[4] Zhenning Cai, Yuwei Fan, and Ruo Li. A framework on moment model reduction for kinetic equation. SIAM Journal on Applied Mathematics, 75(5):2001-2023, 2015.

[5] Manuel Castro, Jorge Macías, and Carlos Parés. A q-scheme for a class of systems of coupled conservation laws with source term. application to a two-layer 1-d shallow water system. ESAIM: Mathematical Modelling and Numerical Analysis, 35(1):107-127, 2001.

[6] Manuel Castro, Philippe G. LeFloch, Maria Luz Munoz-Ruiz, and Carlos Pares. Why many theories of shock waves are necessary: Convergence error in formally path-consistent schemes. Journal of Computational Physics, 227(17):8107-8129, 2008.

[7] Marc Christen, Julia Kowalski, and Perry Bartelt. Ramms: Numerical simulation of dense snow avalanches in three-dimensional terrain. Cold Regions Science and Technology, 63(1-2):114, 2010.

[8] Philippe Courtier and Jean-Francois Geleyn. A global numerical weather prediction model with variable resolution: Application to the shallow-water equations. Quarterly Journal of the Royal Meteorological Society, 114(483):1321-1346, 1988.

[9] Richard V. Craster and Omar K. Matar. Dynamics and stability of thin liquid films. Reviews of modern physics, 81(3):1131, 2009.

[10] G. Dal Maso, P. G. LeFloch, and F. Murat. Definition and weak stability of nonconservative products. Journal de Mathmatiques Pures et Appliques, 74(6):483-548, 1995.

[11] Yana Di, Yuwei Fan, Ruo Li, and Lingchao Zheng. Linear stability of hyperbolic moment models for boltzmann equation. Numerical Mathematics: Theory, Methods and Applications, 10:255-277, 2017. 
[12] Lawrence C. Evans. Partial Differential Equations. Graduate studies in mathematics. American Mathematical Society, 1998.

[13] Yuwei Fan, Julian Koellermeier, Jun Li, Ruo Li, and Manuel Torrilhon. Model reduction of kinetic equations by operator projection. Journal of Statistical Physics, 162(2):457-486, 2016.

[14] Enrique D. Fernández-Nieto, Manuel Castro, and Carlos Parés. On an intermediate field capturing riemann solver based on a parabolic viscosity matrix for the two-layer shallow water system. Journal of Scientific Computing, 48(1-3):117-140, 2011.

[15] Haitham K. Ghamry and Peter M. Steffler. Two dimensional vertically averaged and moment equations for rapidly varied flows. Journal of Hydraulic Research, 40(5):579-587, 2002.

[16] Harold Grad. On the kinetic theory of rarefied gases. Communications on Pure and Applied Mathematics, 2(4):331-407, 1949.

[17] Mohit Gupta and Srinivasa G. Narasimhan. Legendre polynomials triple product integral and lower-degree approximation of polynomials using chebyshev polynomials. Technical Report CMU-RI-TR-07-22, Carnegie Mellon University, Pittsburgh, PA, 2007.

[18] Frank M. Henderson. Open channel flow. Macmillan series in civil engineering. Macmillan, 1966.

[19] Columban Hutter, Yongqi WangShiva, Shiva P. Pudasaini. The savagehutter avalanche model: how far can it be pushed? Philosophical Transactions of the Royal Society of London A: Mathematical, Physical and Engineering Sciences, 363:15071528, 2005.

[20] Martin A. Kern, Perry A. Bartelt, and Betty Sovilla. Velocity profile inversion in dense avalanche flow. Annals of Glaciology, 51(54):2731, 2010.

[21] Julian Koellermeier and ManuelTorrilhon. Numerical study of partially conservative moment equations in kinetic theory. Communications in Computational Physics, 21(04)(4):9811011, 2017.

[22] Julian Koellermeier. Derivation and numerical solution of hyperbolic moment equations for rarefied gas flows. Dissertation, RWTH Aachen University, Aachen, 2017.

[23] Julian Koellermeier, Roman P. Schaerer, and Manuel Torrilhon. A framework for hyperbolic approximation of kinetic equations using quadrature-based projection methods. Kinetic and Related Models, 7(3):531-549, 2014.

[24] Julian Koellermeier and Manuel Torrilhon. Hyperbolic moment equations using quadrature-based projection methods. In AIP Conference Proceedings, volume 1628, pages 626-633. AIP, 2014.

[25] Julia Kowalski. Two-phase modeling of debris flows. PhD thesis, 2008.

[26] Julia Kowalski and Jim N. McElwaine. Shallow two-component gravity-driven flows with vertical variation. Journal of Fluid Mechanics, 714:434-462, 2013.

[27] Julia Kowalski and Manuel Torrilhon. Moment approximations and model cascades for shallow flow. Communications in Computational Physics, 25, 2019.

[28] Nino Krvavica, Miran Tuhtan, and Gordan Jelenić. Analytical implementation of roe solver for two-layer shallow water equations with accurate treatment for loss of hyperbolicity. $A d-$ vances in Water Resources, 122:187-205, 2018.

[29] Pierre-Yves Lagrée, Guillaume Saingier, Stéphanie Deboeuf, Lydie Staron, and Stéphane Popinet. Granular front for flow down a rough incline: about the value of the shape factor in depths averaged models. In EPJ Web of Conferences, volume 140, page 03046. EDP Sciences, 2017.

[30] Randall J. LeVeque. Finite Volume Methods for Hyperbolic Problems. Cambridge Texts in Applied Mathematics. Cambridge University Press, 2002.

[31] David Levermore. Moment closure hierarchies for kinetic theories. Journal of Statistical 
Physics, 83(5):1021-1065, 1996.

[32] Edie Miglio, Alfio Quarteroni, and Fausto Saleri. Finite element approximation of quasi-3d shallow water equations. Computer methods in applied mechanics and engineering, 174(3-4):355369, 1999.

[33] Nicoletta Sanvitale and Elisabeth T. Bowman. Using piv to measure granular temperature in saturated unsteady polydisperse granular flows. Granular Matter, 18(3):57, 2016.

[34] Roman Pascal Schaerer and Manuel Torrilhon. On singular closures for the 5-moment system in kinetic gas theory. Communications in Computational Physics, 17(2):371-400, 2015.

[35] J. B. Schijf and Johan C. Schönfled. Theoretical considerations on the motion of salt and fresh water. In Minnesota International Hydraulic Convention. IAHR, 1953.

[36] Guglielmo Stecca, Annunziato Siviglia, and Eleuterio F. Toro. Upwind-biased FORCE schemes with applications to free-surface shallow flows. Journal of Computational Physics, 229(18):6362-6380, September 2010.

[37] Peter M. Steffler and Jin Yee-Chung. Depth averaged and moment equations for moderately shallow free surface flow. Journal of Hydraulic Research, 31(1):5-17, 1993.

[38] Manuel Torrilhon. Hyperbolic moment equations in kinetic gas theory based on multivariate Pearson-IV-distributions. Communications in Computational Physics, 7(4):639-673, 2010.

[39] Yanli Wang and Shudao Zhang. Solving vlasov-poisson-fokker-planck equations using nrxx method. Communications in Computational Physics, 21:782-807, 2017. 